\title{
$A-\phi$ formulation of a mathematical model for the induction hardening process with a nonlinear law for the magnetic field
}

\author{
Jaroslav Chovan ${ }^{\mathrm{a}}$, Christophe Geuzaine ${ }^{\mathrm{b}}$, Marián Slodička ${ }^{\mathrm{a}, *}$ \\ a Department of Mathematical Analysis, Ghent University, Ghent, Belgium \\ ${ }^{\mathrm{b}}$ Department of Electrical Engineering and Computer Science, Montefiore Institute, University of Liege, Liege, Belgium
}

Received 28 October 2016; received in revised form 2 March 2017; accepted 30 March 2017

Available online 25 April 2017

\begin{abstract}
We derive and analyze a mathematical model for induction hardening. We assume a nonlinear relation between the magnetic field and the magnetic induction field. For the electromagnetic part, we use the vector-scalar potential formulation.

The coupling between the electromagnetic and the thermal part is provided through the temperature-dependent electric conductivity and the joule heating term, the most crucial element, considering the mathematical analysis of the model. It acts as a source of heat in the thermal part and leads to the increase in temperature. Therefore, in order to be able to control it, we apply a truncation function.

Using Rothe's method, we prove the existence of a global solution to the whole system. The nonlinearity in the electromagnetic part is handled by the theory of monotone operators. To supplement our theoretical results we provide a numerical simulation using real physical constants.
\end{abstract}

(c) 2017 Elsevier B.V. All rights reserved.

Keywords: Maxwell's equations; Minty-Browder; Monotone operators; Rothe's method; Scalar potential; Vector potential

\section{Introduction}

There are many papers dealing with mathematical models of the induction hardening process. Some of them provide various numerical schemes e.g. [1-6]. But they omit mathematical or numerical analysis of their models and numerical schemes. Other papers deal with the well-posedness of the problem and provide theoretical results e.g. [7-11]. The topic of induction hardening has been broadly covered in papers [12,13] and [14]. However, all manuscripts tackling the theoretical side of the induction hardening phenomena present mathematical models with linear dependency between magnetic and magnetic induction field. The papers $[15,16]$ studied a mathematical model

* Corresponding author.

E-mail addresses: jaroslav.chovan@ugent.be (J. Chovan), cgeuzaine@ulg.ac.be (C. Geuzaine), marian.slodicka@ugent.be (M. Slodička).

URLs: http://cage.ugent.be/ jchovan (J. Chovan), http://montefiore.ulg.ac.be/ qgeuzaine (C. Geuzaine), http://cage.ugent.be/ ms (M. Slodička). 


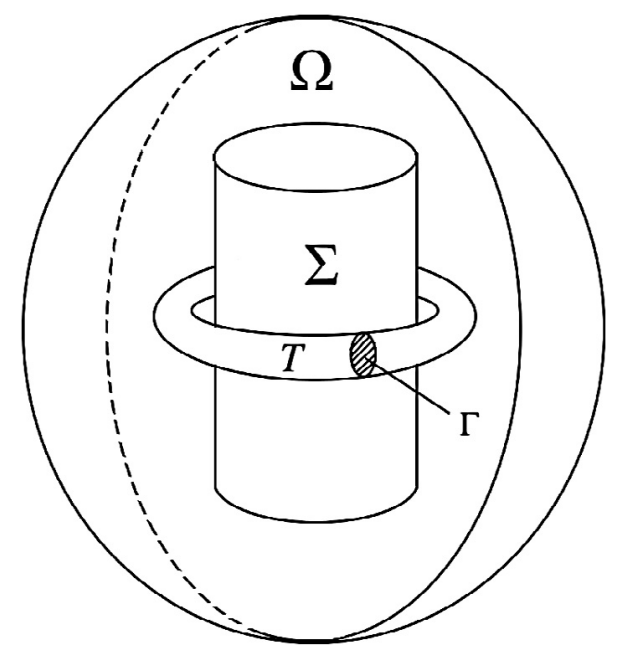

Fig. 1. Illustration of the domain.

with a nonlinear relation between those two vectorial fields (which better reflects the reality), but the study was restricted just to a conductor, i.e. the domain had only one component. The authors proved solvability for a formulation with either magnetic induction or magnetic field as unknown. We present the vector-scalar potential formulation for a nonlinear setting including conducting and non-conducting parts, i.e. the domain consists of multiple components. This means that material coefficients may have jumps across the interfaces. To our best knowledge nothing similar has been done before.

\subsection{Derivation of a mathematical model}

We work only with a simplified model of induction hardening process (see Fig. 1). The time frame is denoted by $[0, \mathcal{T}]$. Let $\Omega$ be a bounded sphere in $\mathbb{R}^{3}$. The workpiece and the coil are represented by $\Sigma$ and $T$, respectively. Both $\Sigma$ and $T$ are closed subsets of $\Omega$ and the following holds

$$
\Sigma \cap T=\varnothing, \text { and } \partial \Sigma, \partial T, \partial \Omega \text { are of class } C^{1,1} .
$$

Conductors are affected by temperature, hence we separate them from the rest of the domain $\Omega$ by denoting $\pi=\Sigma \cup T$. Current in the coil is modeled via an interface condition on $\Gamma$. By $\boldsymbol{v}$ we denote the standard outer normal unit vector associated with surfaces of materials under consideration.

We start deriving our mathematical model with introducing the classical Maxwell equations (for reference, see [17])

$$
\begin{aligned}
& \nabla \cdot \boldsymbol{D}=\rho, \\
& \nabla \cdot \boldsymbol{B}=0, \\
& \nabla \times \boldsymbol{E}=-\partial_{t} \boldsymbol{B}, \\
& \nabla \times \boldsymbol{H}=\partial_{t} \boldsymbol{D}+\boldsymbol{J} .
\end{aligned}
$$

Here, $\boldsymbol{D}$ stands for displacement current and $\rho$ is the density of electrical charge. The magnetic induction field, the electrical field and the magnetic field are denoted with $\boldsymbol{B}, \boldsymbol{E}$ and $\boldsymbol{H}$, respectively. At last, $\boldsymbol{J}$ indicates the source current. For the clarity, we note that equations above are true in the whole domain $\Omega$.

In models dealing with eddy currents, the time variation of displacement current is insignificant, therefore we neglect it. We present the nonlinear relation between $\boldsymbol{H}$ and $\boldsymbol{B}$ in the following form:

$$
\boldsymbol{H}:=\mu \boldsymbol{M}(\boldsymbol{B})=\frac{1}{\mu^{*}} m(|\boldsymbol{B}|) \boldsymbol{B} .
$$


Magnetic permeability $\mu=\frac{1}{\mu^{*}}$ might behave differently in the workpiece and in the air, therefore, we specify it as a split function

$$
\mu(\boldsymbol{x})= \begin{cases}\mu_{\pi}(\boldsymbol{x}), & \text { if } \boldsymbol{x} \in \bar{\pi} \\ \mu_{A}(\boldsymbol{x}), & \text { if } \boldsymbol{x} \in \Omega \backslash \bar{\pi}\end{cases}
$$

Both $\mu_{\pi}$ and $\mu_{A}$ are strictly positive and bounded. There is no jump in the tangential component of $\boldsymbol{H}$ along the boundaries between different materials, i.e.

$$
[\mu \boldsymbol{M}(\nabla \times \boldsymbol{A}) \times \boldsymbol{v}]_{\partial \pi}=\mathbf{0} .
$$

The vectorial field $\boldsymbol{M}$ is supposed to be potential and its potential is denoted $\Phi_{\boldsymbol{M}}$, i.e. $\nabla \Phi_{\boldsymbol{M}}=\boldsymbol{M}$, cf. [18]. Moreover, we assume that $\boldsymbol{M}$ is strictly monotone and Lipschitz continuous. Furthermore, we introduce Ohm's law

$$
\boldsymbol{J}=\sigma \boldsymbol{E} .
$$

Function $\sigma$ represents the electric conductivity and it is defined as follows

$$
\sigma(u(\boldsymbol{x}, t))= \begin{cases}\sigma_{\pi}(u(\boldsymbol{x}, t)), & \text { if } \boldsymbol{x} \in \overline{\boldsymbol{\pi}}, t \in[0, \mathcal{T}], \\ 0, & \text { if } \boldsymbol{x} \in \Omega \backslash \overline{\boldsymbol{\pi}}, t \in[0, \mathcal{T}],\end{cases}
$$

where $u(\boldsymbol{x}, t)$ is a function of temperature in the workpiece and the coil. We consider $\sigma$ to be continuous, bounded and strictly positive in $\overline{\boldsymbol{\pi}}$. Since $\Omega$ is a simply-connected domain and (3) is true in the whole $\Omega$, we use ([19, Theorem 3.6]) to obtain exactly one magnetic vector potential $\boldsymbol{A} \in \mathbf{H}(\mathbf{c u r l} ; \Omega)$ with the following properties:

$$
\boldsymbol{B}=\nabla \times \boldsymbol{A}, \quad \nabla \cdot \boldsymbol{A}=0, \quad \boldsymbol{A} \times \boldsymbol{v}=\mathbf{0} \text { on } \partial \Omega .
$$

Substituting (10) into (4) we get

$$
\nabla \times\left(\boldsymbol{E}+\partial_{t} \boldsymbol{A}\right)=\mathbf{0} \text { in } \Omega .
$$

Using (11), we apply ([19, Theorem 2.9]) to acquire a unique scalar potential $\phi \in H^{1}(\Omega) / \mathbb{R}$ such that:

$$
\boldsymbol{E}+\partial_{t} \boldsymbol{A}=-\nabla \phi
$$

Taking into account the insignificance of $\partial_{t} \boldsymbol{D}$ and using (12), (10), (8), (6), (5) we arrive at the following boundary value problem for vector potential $A$ :

$$
\begin{array}{ll}
\sigma \partial_{t} \boldsymbol{A}+\nabla \times \mu \boldsymbol{M}(\nabla \times \boldsymbol{A})+\sigma \chi_{T} \nabla \phi=0 & \text { for a.e. }(\boldsymbol{x}, t) \in \Omega \times(0, \mathcal{T}):=Q_{\mathcal{T}} \\
\boldsymbol{A} \times \boldsymbol{v}=\mathbf{0} & \text { for a.e. }(\boldsymbol{x}, t) \in \partial \Omega \times(0, \mathcal{T}), \\
\boldsymbol{A}(0)=\boldsymbol{A}_{0} & \text { for } \boldsymbol{x} \in \Omega, t=0
\end{array}
$$

Characteristic function $\chi_{T}$ has value 1 , if $\boldsymbol{x} \in T$ and 0 otherwise. We use it, because the external source of the current, which is defined by the gradient of the scalar potential, is present only in the coil ( $T$, see Fig. 1).

Combination of (8) and (12) gives us an expression for the total current density $\boldsymbol{J}$

$$
\boldsymbol{J}=-\sigma \partial_{t} \boldsymbol{A}-\sigma \nabla \phi .
$$

The impressed part $\boldsymbol{J}_{\text {source }}=-\sigma \nabla \phi$ is caused by an external source and the induced part $\boldsymbol{J}_{\text {induced }}=-\sigma \partial_{t} \boldsymbol{A}$ is caused by the magnetic induction field $\boldsymbol{B}$ in the coil. Demanding that the continuity equation holds for the source current $\boldsymbol{J}_{\text {source }}$, i.e.

$$
\nabla \cdot \boldsymbol{J}_{\text {source }}=0
$$

we define the scalar potential $\phi$ by the following elliptic equation with homogeneous Neumann boundary condition on $\partial T$ and interface condition on $\Gamma$, cf. [12]:

$$
\begin{array}{ll}
-\nabla \cdot\left(\sigma_{\pi} \nabla \phi\right)=0 & \text { for a.e. }(\boldsymbol{x}, t) \in T \times(0, \mathcal{T}), \\
-\sigma_{\pi} \frac{\partial \phi}{\partial \boldsymbol{v}}=0 & \text { for a.e. }(\boldsymbol{x}, t) \in \partial T \times(0, \mathcal{T}), \\
{\left[-\sigma_{\pi} \frac{\partial \phi}{\partial \boldsymbol{v}}\right]_{\Gamma}=j} & \text { for a.e. }(\boldsymbol{x}, t) \in \Gamma \times(0, \mathcal{T}) .
\end{array}
$$


External source current density is represented by function $j(x, t)$, which is assumed to be Lipschitz continuous in time. Jump across interface $\Gamma$ is indicated by $[\cdot]_{\Gamma}$.

Eddy currents generated in the workpiece raise temperature by a significant amount. This phenomenon is called Joule heat and it is expressed as

$$
\boldsymbol{J} \cdot \boldsymbol{E} \stackrel{(8)}{=} \sigma_{\pi}|\boldsymbol{E}|^{2} \stackrel{(12)}{=} \sigma_{\pi}\left|\partial_{t} \boldsymbol{A}+\chi_{T} \nabla \phi\right|^{2} .
$$

This term is crucial and causes numerous troubles during mathematical treatment (unboundedness). Therefore, we introduce a cut-off function and work with truncated Joule-heating term

$$
\mathcal{R}_{r}(x):=\left\{\begin{aligned}
r>0 & \text { if } x>r, \\
x & \text { if }|x| \leqslant r, \\
-r & \text { if } x<-r .
\end{aligned}\right.
$$

Evolution of temperature in the workpiece and the coil ( $\pi$, see Fig. 1) is characterized by the following parabolic nonlinear equation with the homogeneous Neumann boundary condition:

$$
\begin{array}{ll}
\partial_{t} \beta(u)-\nabla \cdot(\lambda \nabla u)=\mathcal{R}_{r}\left(\sigma_{\pi}(u)\left|\partial_{t} \boldsymbol{A}+\chi_{T} \nabla \phi\right|^{2}\right) & \text { for a.e. }(\boldsymbol{x}, t) \in \boldsymbol{\pi} \times(0, \mathcal{T}), \\
-\lambda \frac{\partial u}{\partial \boldsymbol{v}}=0 & \text { for a.e. }(\boldsymbol{x}, t) \in \partial \boldsymbol{\pi} \times(0, \mathcal{T}), \\
u(0)=u_{0} & \text { for } \boldsymbol{x} \in \boldsymbol{\pi}, t=0 .
\end{array}
$$

Continuous function $\lambda(\boldsymbol{x}, t)$ is supposed to be strictly positive and bounded. The nonlinear function $\beta$ is of a linear growth and its derivative is bounded from below by a positive constant.

Eqs. (13), (14) and (17) model the process of induction hardening in our simplified domain $\Omega$. They are tied together through terms $\nabla \phi, \sigma$ and $\partial_{t} \boldsymbol{A}$. One could ask, whether the artificial intervention in the form of cut-off function was correct. In real applications of induction hardening, there is always a switch-off button, which is used to prevent the workpiece from thermal deformations. When the temperature reaches a certain degree, this button is turned-off, the stream of electric current is stopped and the workpiece is cooled down. Therefore, applying the cut-off function on Joule-heating term in (17), is actually a simulation of this switch-off button and indeed, necessary to be done.

\section{Functional setting}

\subsection{Variational formulation}

Let us start with some basic notations. Through the whole paper we adopt notation $(\cdot, \cdot)_{\Omega}$ for the standard inner product in $\mathrm{L}^{2}(\Omega)$ or $\mathbf{L}^{2}(\Omega)$. Norm induced by this inner product is indicated as $\|\cdot\|_{\mathrm{L}^{2}(\Omega)}$. Set of functions $k:[0, \mathcal{T}] \rightarrow Y$ equipped with the norm $\max _{t \in[0, \mathcal{T}]}\|\cdot\|_{Y}$ is denoted as $C([0, \mathcal{T}] ; Y)$. In a case when $p>1$, norm in $L^{p}((0, \mathcal{T}) ; Y)$ is defined as $\left(\int_{0}^{\mathcal{T}}\|\cdot\|_{Y}^{p} \mathrm{~d} t\right)^{\frac{1}{p}}$. Set of all functions $\phi+c$, where $\phi \in H^{1}(T)$ and $c$ is a constant is marked as $\phi_{c}$.

Considering the vector potential $\boldsymbol{A}$, we introduce the Hilbert space

$$
\boldsymbol{X}_{N, 0}=\{\boldsymbol{\varphi} \in \mathbf{H}(\mathbf{c u r l} ; \Omega) ; \nabla \cdot \boldsymbol{\varphi}=0 \text {, and } \boldsymbol{\varphi} \times \boldsymbol{v}=0 \text { on } \partial \Omega\},
$$

where $\mathbf{H}($ curl $; \Omega)=\left\{\boldsymbol{\varphi} \in \mathbf{L}^{2}(\Omega): \nabla \times \boldsymbol{\varphi} \in \mathbf{L}^{2}(\Omega)\right\}$. Using Friedrichs' inequality for vectorial fields (cf. [19, Lemma 3.4] or [20, Cor. 3.51]) we see that we may furnish $\boldsymbol{X}_{N, 0}$ with norm $\|\boldsymbol{\varphi}\|_{\boldsymbol{X}_{N, 0}}:=\|\nabla \times \varphi\|_{\mathbf{L}^{2}(\Omega)}$. Taking into account (1), we use [19, Theorem 3.7] or [21, Theorem 2.12] to conclude that $\boldsymbol{X}_{N, 0}$ is a closed subspace of $\mathbf{H}^{1}(\Omega){ }^{1}$ Multiplying (13) by a test function $\boldsymbol{\varphi} \in \boldsymbol{X}_{N, 0}$, integrating over $\Omega$ and using Green's theorem, we obtain the

\footnotetext{
${ }^{1}$ The relation $\boldsymbol{X}_{N, 0} \subset \mathbf{H}^{1}(\Omega)$ is crucial for our mathematical approach. We would like to point out that the same inclusion is valid also for convex domains (with non smooth boundary). In such a case one can rely on the [21, Theorem 2.17]. All presented results hold true also for convex domains.
} 


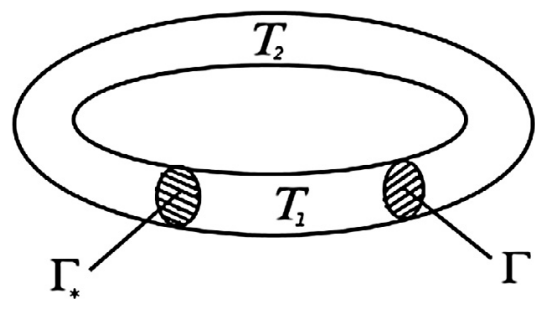

Fig. 2. Dissection of $T$.

variational formulation for vector potential $\boldsymbol{A}$ :

$$
\left(\sigma_{\pi} \partial_{t} \boldsymbol{A}, \boldsymbol{\varphi}\right)_{\pi}+(\mu \boldsymbol{M}(\nabla \times \boldsymbol{A}), \nabla \times \boldsymbol{\varphi})_{\Omega}+\left(\sigma_{\pi} \nabla \phi, \boldsymbol{\varphi}\right)_{T}=0 \quad \forall \boldsymbol{\varphi} \in \boldsymbol{X}_{N, 0} .
$$

To obtain the variational formulation for (14), we split $T$ in two separate parts $T_{1}$ and $T_{2}$. Flux of the scalar potential on the new interface $\Gamma_{*}$ is supposed to be continuous. Moreover, $\Gamma_{*} \cap \Gamma=\emptyset$ and $T_{1} \cap T_{2}=\Gamma_{*} \cup \Gamma$ (see Fig. 2). Now, we multiply (14) by a test function $\xi \in H^{1}(T) / \mathbb{R}$ and integrate in $T_{1}$ and $T_{2}$. Using Green's theorem, boundary condition (14) and continuous condition on $\Gamma_{*}$, we arrive to the following variational formulation for scalar potential $\phi$ :

$$
\left(\sigma_{\pi} \nabla \phi, \nabla \xi\right)_{T}+(j, \xi)_{\Gamma}=0 \quad \forall \xi \in H^{1}(T) / \mathbb{R} .
$$

The choice of the test space $H^{1}(T) / \mathbb{R}$ is just to obtain a unique solvability.

Lemma 1. There are positive constants $c_{1}$ and $c_{2}$ such that:

$$
c_{1}\left\|\phi_{c}\right\|_{H^{1}(T) / \mathbb{R}}^{2} \leqslant\|\nabla \phi\|_{\mathbf{L}^{2}(T)}^{2} \leqslant c_{2}\left\|\phi_{c}\right\|_{H^{1}(T) / \mathbb{R}}^{2} .
$$

Proof. Norm in $H^{1}(T) / \mathbb{R}$ is defined as $\left\|\phi_{c}\right\|_{H^{1}(T) / \mathbb{R}}:=\inf _{\phi \in \phi_{c}}\|\phi\|_{H^{1}(T)}$. This norm is minimal for $c=-\frac{1}{|T|} \int_{T} \phi \mathrm{d} x$. Indeed, let us take a closer look.

$$
0=\frac{d}{d c}\left(\int_{T}(\phi+c)^{2}+|\nabla \phi|^{2} \mathrm{~d} x\right)=2 \int_{T} \phi \mathrm{d} x+2 \int_{T} c \mathrm{~d} x \Longrightarrow c=-\frac{1}{|T|} \int_{T} \phi \mathrm{d} x .
$$

Now, we write $\left\|\phi_{c}\right\|_{H^{1}(T) / \mathbb{R}}=\left\|\phi-\frac{1}{|T|} \int_{T} \phi \mathrm{d} x\right\|_{H^{1}(T)}$. Using Poincaré-Wirtinger inequality, cf. [22] we conclude the following:

$$
\begin{aligned}
\left\|\phi_{c}\right\|_{H^{1}(T) / \mathbb{R}}^{2} & =\left\|\phi-\frac{1}{|T|} \int_{T} \phi \mathrm{d} x\right\|_{L^{2}(T)}^{2}+\|\nabla \phi\|_{\mathbf{L}^{2}(T)}^{2} \leqslant c_{P W}\|\nabla \phi\|_{\mathbf{L}^{2}(T)}^{2}+\|\nabla \phi\|_{\mathbf{L}^{2}(T)}^{2} \\
& =\left(c_{P W}+1\right)\|\nabla \phi\|_{\mathbf{L}^{2}(T)}^{2},
\end{aligned}
$$

where $c_{P W}$ is a positive constant. Taking $c_{2}=1$ and $c_{1}=\frac{1}{1+c_{P W}}$, the proof is completed.

For Eq. (17) we follow identical steps as above, using $\psi \in H^{1}(\pi)$ as a test function, which brings us to the variational formulation for function $u$ :

$$
\left(\partial_{t} \beta(u), \psi\right)_{\pi}+(\lambda \nabla u, \nabla \psi)_{\pi}=\left(\mathcal{R}_{r}\left(\sigma_{\pi}\left|\partial_{t} \boldsymbol{A}+\chi_{T} \nabla \phi\right|^{2}\right), \psi\right)_{\pi} \quad \forall \psi \in H^{1}(\pi) .
$$

Norm in $H^{1}(\pi)$ is defined as $\|\psi\|_{H^{1}(\pi)}^{2}:=\|\psi\|_{L^{2}(\pi)}^{2}+\|\nabla \psi\|_{\mathbf{L}^{2}(\pi)}^{2}$. 


\subsection{Assumptions}

To achieve better clarity and readability of our paper, we list all assumptions altogether:

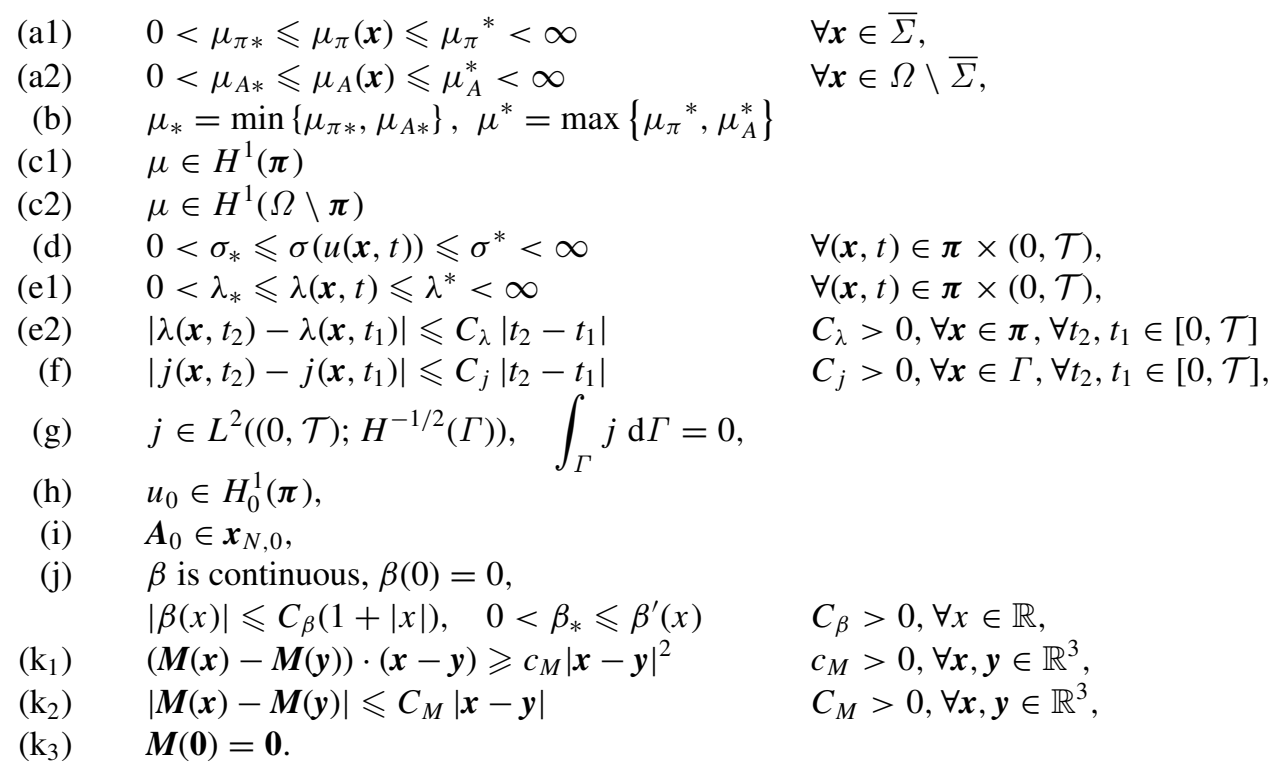

Following [18, Theorem 5.1], we see that potential $\Phi_{M}$ of vectorial field $\boldsymbol{M}$ with properties $\left(\mathrm{k}_{1}\right)-\left(\mathrm{k}_{3}\right)$, is strictly convex. Applying [18, Theorem 8.4], we get

$$
\boldsymbol{M}(\boldsymbol{x}) \cdot(\boldsymbol{x}-\boldsymbol{y}) \geqslant \Phi_{\boldsymbol{M}}(\boldsymbol{x})-\Phi_{\boldsymbol{M}}(\boldsymbol{y}) \quad \forall \boldsymbol{x}, \boldsymbol{y} \in \mathbb{R}^{3} .
$$

Thanks to $\left(\mathrm{k}_{1}\right)$ and $\left(\mathrm{k}_{2}\right)$, we bound $\Phi_{M}$ from below

$$
\begin{aligned}
\Phi_{\boldsymbol{M}}(\boldsymbol{x}) & =\int_{0}^{1} \boldsymbol{M}(\boldsymbol{x} p) \cdot \boldsymbol{x} \mathrm{d} p=\int_{0}^{1} \boldsymbol{M}(\boldsymbol{x} p) \cdot(\boldsymbol{x} p) p^{-1} \mathrm{~d} p \\
& \geqslant \int_{0}^{1} c_{M}|\boldsymbol{x} p|^{2} p^{-1} \mathrm{~d} p=\frac{c_{M}}{2}|\boldsymbol{x}|^{2} .
\end{aligned}
$$

We get

$$
\Phi_{M}(x) \leqslant \frac{C_{M}}{2}|x|^{2}
$$

from $\left(\mathrm{k}_{2}\right)$ in the same way.

\section{Existence of a weak solution}

\subsection{Time discretization scheme and a priori estimates}

In this section we discretize the time interval $[0, \mathcal{T}]$ and solve a system of steady-state differential equations on each time step. Afterwards, we construct piece-wise constant and piece-wise linear in time functions and show convergence of sub-sequences of these functions in appropriate functional spaces to the weak solution. This approach is called Rothe's method $[23,24]$. Consider a number of time steps $n \in \mathbb{N}$. We introduce a time discretization of $[0, \mathcal{T}]$ in the following sense:

$$
[0, \mathcal{T}]=\bigcup_{0 \leqslant i \leqslant n-1}\left[t_{i}, t_{i-1}\right], \text { where } t_{i}=i \tau, 0 \leqslant i \leqslant n, n \tau=\mathcal{T} .
$$

The value of any function $f$ at $t_{i}$ is denoted as $f_{i}$. To approximate the time derivative of $f$ at $t_{i}$ we use the explicit Euler method, i.e.

$$
\partial_{t} f\left(t_{i}\right):=\delta f_{i}=\frac{f_{i}-f_{i-1}}{\tau} .
$$


Applying this method to the system (18), (20), (19) we are able to approximate it at every time step $t_{i}$, for $i=$ $1, \ldots, n$

$$
\begin{aligned}
\left(\sigma_{\pi}\left(u_{i-1}\right) \nabla \phi_{c_{i}}, \nabla \xi\right)_{T}+\left(j_{i}, \xi\right)_{\Gamma}=0 & \text { for any } \quad \xi \in H^{1}(T) / \mathbb{R}, \\
\left(\sigma_{\pi}\left(u_{i-1}\right) \delta \boldsymbol{A}_{i}, \boldsymbol{\varphi}\right)_{\pi}+\left(\mu \boldsymbol{M}\left(\nabla \times \boldsymbol{A}_{i}\right), \nabla \times \boldsymbol{\varphi}\right)_{\Omega}+\left(\sigma_{\pi}\left(u_{i-1}\right) \nabla \phi_{c_{i}}, \boldsymbol{\varphi}\right)_{T}=0 & \text { for any } \boldsymbol{\varphi} \in \boldsymbol{X}_{N, 0}, \\
\left(\delta \beta\left(u_{i}\right), \psi\right)_{\pi}+\left(\lambda_{i} \nabla u_{i}, \psi\right)_{\pi}-\left(\mathcal{R}_{r}\left(\sigma_{\pi}\left(u_{i-1}\right)\left|\delta \boldsymbol{A}_{i}+\chi_{T} \nabla \phi_{c_{i}}\right|^{2}\right), \psi\right)_{\pi}=0 & \text { for any } \quad \psi \in H^{1}(\boldsymbol{\pi}) .
\end{aligned}
$$

Remark 1. In system (25)-(27) we use $u_{i-1}$ as an argument for function $\sigma$. The reason to take this action is to be able to decouple the whole system. As we will see in the sequel, this small adjustment does not affect convergence results.

To prove the solvability at each time step we use the theory of monotone operators (for more details, see [18,25]).

Lemma 2. Assume that (21) holds. Then, for any $i=1, \ldots, n$, there exists a uniquely determined triplet $\phi_{c_{i}} \in H^{1}(T) / \mathbb{R}, \boldsymbol{A}_{i} \in X_{N, 0}$ and $u_{i} \in H^{1}(\boldsymbol{\pi})$ solving system (25)-(27).

Proof. Let us define operators: $\mathcal{F}_{\sigma}: \boldsymbol{X}_{N, 0} \rightarrow\left(\boldsymbol{X}_{N, 0}\right)^{*}$ and $\mathcal{G}_{\lambda}: H^{1}(\boldsymbol{\pi}) \rightarrow\left(H^{1}(\boldsymbol{\pi})\right)^{*}$

$$
\begin{aligned}
\left\langle\mathcal{F}_{\sigma}(\boldsymbol{A}), \boldsymbol{\varphi}\right\rangle & :=\left(\sigma \frac{\boldsymbol{A}}{\tau}, \boldsymbol{\varphi}\right)_{\pi}+(\mu \boldsymbol{M}(\nabla \times \boldsymbol{A}), \nabla \times \boldsymbol{\varphi})_{\Omega}, \\
\left\langle\mathcal{G}_{\lambda}(u), \psi\right\rangle & :=\left(\frac{\beta(u)}{\tau}, \psi\right)_{\pi}+(\lambda \nabla u, \nabla \psi)_{\pi} .
\end{aligned}
$$

We need to show that these operators are hemicontinuous, strictly monotone and coercive.

Hemicontinuity follows from continuity of $\boldsymbol{M}$ and $\beta$. To show the strict monotonicity of the first operator we use the strongly monotone character of $\boldsymbol{M}$ (which also implies strict monotonicity). We write for some positive constant $C$ and $\tau \in(0,1)$

$$
\begin{aligned}
\left\langle\mathcal{F}_{\sigma}\left(\boldsymbol{A}_{1}\right)-\mathcal{F}_{\sigma}\left(\boldsymbol{A}_{2}\right), \boldsymbol{A}_{1}-\boldsymbol{A}_{2}\right\rangle= & \left(\frac{\sigma}{\tau}\left(\boldsymbol{A}_{1}-\boldsymbol{A}_{2}\right), \boldsymbol{A}_{1}-\boldsymbol{A}_{2}\right)_{\pi} \\
& +\left(\mu\left(\boldsymbol{M}\left(\nabla \times \boldsymbol{A}_{1}\right)-\boldsymbol{M}\left(\nabla \times \boldsymbol{A}_{2}\right)\right), \nabla \times\left(\boldsymbol{A}_{1}-\boldsymbol{A}_{2}\right)\right)_{\Omega} \\
\geqslant & \frac{\sigma_{*}}{\tau}\left\|\boldsymbol{A}_{1}-\boldsymbol{A}_{2}\right\|_{\mathbf{L}^{2}(\pi)}^{2}+\mu_{*}\left\|\nabla \times\left(\boldsymbol{A}_{1}-\boldsymbol{A}_{2}\right)\right\|_{\mathbf{L}^{2}(\Omega)}^{2} \\
\geqslant & C \boldsymbol{A}_{1}-\boldsymbol{A}_{2} \|_{\boldsymbol{x}_{N, 0}}^{2}>0
\end{aligned}
$$

for any $\boldsymbol{A}_{1}, \boldsymbol{A}_{2} \in \boldsymbol{X}_{N, 0}, \boldsymbol{A}_{1} \neq \boldsymbol{A}_{2}$. In other words the operator $\mathcal{F}_{\sigma}$ is strictly monotone. To show that also $\mathcal{G}_{\lambda}$ is strictly monotone we take into account the properties of scalar potential $\beta$ and use the mean value theorem. Then we write for $\tau \in(0,1)$ and for some positive constants $C$ and $\eta \in(0,1)$

$$
\begin{aligned}
\left\langle\mathcal{G}_{\lambda}\left(u_{1}\right)-\mathcal{G}_{\lambda}\left(u_{2}\right), u_{1}-u_{2}\right\rangle & =\left(\frac{\beta^{\prime}\left[u_{1}+\eta\left(u_{2}-u_{1}\right)\right]}{\tau},\left|u_{1}-u_{2}\right|^{2}\right)_{\pi}+\left(\lambda\left(\nabla u_{1}-\nabla u_{2}\right), \nabla u_{1}-\nabla u_{2}\right)_{\pi} \\
& \geqslant \frac{\beta_{*}}{\tau}\left\|u_{1}-u_{2}\right\|_{\mathbf{L}^{2}(\pi)}^{2}+\lambda_{*}\left\|\nabla u_{1}-\nabla u_{2}\right\|_{\mathbf{L}^{2}(\pi)}^{2} \\
& \geqslant C\left\|u_{1}-u_{2}\right\|_{H^{1}(\pi)}^{2}>0
\end{aligned}
$$

for any $u_{1}, u_{2} \in H^{1}(\pi), u_{1} \neq u_{2}$. Coercivity of these operators is guaranteed since $\boldsymbol{M}(\mathbf{0})=\mathbf{0}$ and $\beta(0)=0$. We have

$$
\begin{aligned}
\left\langle\mathcal{F}_{\sigma}(\boldsymbol{A}), \boldsymbol{A}\right\rangle & =\left(\frac{\sigma}{\tau} \boldsymbol{A}, \boldsymbol{A}\right)_{\pi}+(\mu(\boldsymbol{M}(\nabla \times \boldsymbol{A})-\boldsymbol{M}(\mathbf{0})), \nabla \times \boldsymbol{A}-\mathbf{0})_{\Omega} \geqslant C\|\boldsymbol{A}\|_{\boldsymbol{X}_{N, 0}}^{2}, \\
\left\langle\mathcal{G}_{\lambda}(u), u\right\rangle & =\left(\frac{\beta(u)-\beta(0)}{\tau}, u-0\right)_{\pi}+(\lambda \nabla u, \nabla u)_{\pi} \geqslant C\|u\|_{H^{1}(\pi)}^{2} .
\end{aligned}
$$

Thus

$$
\lim _{\|\boldsymbol{A}\|_{\boldsymbol{X}_{N, 0}} \rightarrow \infty} \frac{\left\langle\mathcal{F}_{\sigma}(\boldsymbol{A}), \boldsymbol{A}\right\rangle}{\|\boldsymbol{A}\|_{\boldsymbol{X}_{N, 0}}} \geqslant+\infty \quad \text { and } \quad \lim _{\|u\|_{H^{1}(\pi)} \rightarrow \infty} \frac{\left\langle\mathcal{G}_{\lambda}(u), u\right\rangle}{\|u\|_{H^{1}(\pi)}} \geqslant+\infty .
$$

We have shown the strict monotonicity, coercivity and hemicontinuity of operators $\mathcal{F}_{\sigma}$ and $\mathcal{G}_{\lambda}$. 
Rest of the proof serves as a guideline for obtaining a solution-triplet at every time step $t=t_{i}$, for $i=1, \ldots, n$. Applying Lax-Milgram lemma (see [20, Lemma 2.21]) to (25) we obtain a unique solution $\phi_{c_{i}} \in H^{1}(\boldsymbol{\pi}) / \mathbb{R}$ at a time step $t=t_{i}\left(u_{i-1}\right.$ is known at this time step).

To obtain a unique solution $\boldsymbol{A}_{i}$ at a time step $t_{i}$ we have to solve the following identity:

$$
\left\langle\mathcal{F}_{\sigma_{\pi}\left(u_{i-1}\right)}\left(\boldsymbol{A}_{i}\right), \boldsymbol{\varphi}\right\rangle=\left(\sigma_{\pi}\left(u_{i-1}\right) \frac{\boldsymbol{A}_{i-1}}{\tau}, \boldsymbol{\varphi}\right)_{\pi}-\left(\sigma_{\pi}\left(u_{i-1}\right) \nabla \phi_{c_{i}}, \boldsymbol{\varphi}\right)_{T} .
$$

Since the right-hand side (RHS) is known and the operator $\mathcal{F}_{\sigma_{\pi}\left(u_{i-1}\right)}$ is hemicontinuous, strictly monotone and coercive we use [18, Theorem 18.2] to provide the solution. The basic idea of this theorem is to replace the original equation by finite-dimensional approximate equations and then prove the convergence of this approximation scheme. Such technique is called the Galerkin method. Now, we involve the same theorem again to acquire a unique solution $u_{i} \in H^{1}(\boldsymbol{\pi})$ of the setting below (taking into account that the RHS is known)

$$
\left\langle\mathcal{G}_{\lambda_{i}}\left(u_{i}\right), \psi\right\rangle=\left(\frac{\beta\left(u_{i-1}\right)}{\tau}, \psi\right)_{\pi}+\left(\mathcal{R}_{r}\left(\sigma_{\pi}\left(u_{i-1}\right)\left|\delta \boldsymbol{A}_{i}+\chi_{T} \nabla \phi_{c_{i}}\right|^{2}\right), \psi\right)_{\pi} .
$$

This provides us with the solution-triplet $\left\{\phi_{c_{i}}, \boldsymbol{A}_{i}, u_{i}\right\}$ at a time step $t=t_{i}$, for $i=1, \ldots, n$.

To wrap everything together we state a pseudo-scheme for obtaining the solution-triplet $\left\{\phi_{c_{i}}, \boldsymbol{A}_{i}, u_{i}\right\}$ for every time step $t=t_{i}$ :

1. Let $i$ be given and assume that $u_{i-1}, j_{i}$ and $\lambda_{i}$ are known

2. Find $\phi_{c_{i}}$ from:

$\left(\sigma_{\pi}\left(u_{i-1}\right) \nabla \phi_{c_{i}}, \nabla \xi\right)_{T}+\left(j_{i}, \xi\right)_{\Gamma}=0$

3. Find $\boldsymbol{A}_{i}$ from:

$\left(\sigma_{\pi}\left(u_{i-1}\right) \frac{\boldsymbol{A}_{i}}{\tau}, \boldsymbol{\varphi}\right)_{\pi}+\left(\mu \boldsymbol{M}\left(\nabla \times \boldsymbol{A}_{i}\right), \nabla \times \boldsymbol{\varphi}\right)_{\Omega}=\left(\sigma_{\pi}\left(u_{i-1}\right) \frac{\boldsymbol{A}_{i-1}}{\tau}, \boldsymbol{\varphi}\right)_{\pi}-\left(\sigma_{\pi}\left(u_{i-1}\right) \nabla \phi_{c_{i}}, \boldsymbol{\varphi}\right)_{T}$

4. Find $u_{i}$ from:

$\left(\frac{\beta\left(u_{i}\right)}{\tau}, \psi\right)_{\pi}+\left(\lambda_{i} \nabla u_{i}, \nabla \psi\right)_{\pi}=\left(\frac{\beta\left(u_{i-1}\right)}{\tau}, \psi\right)_{\pi}+\left(\mathcal{R}_{r}\left(\sigma_{\pi}\left(u_{i-1}\right)\left|\delta \boldsymbol{A}_{i}+\chi_{T} \nabla \phi_{c_{i}}\right|^{2}\right), \psi\right)_{\pi}$

5. Set $i=i+1$ and repeat the process.

Before we proceed to the main theorem, we have to derive some basic energy estimates for $\phi_{c_{i}}, \boldsymbol{A}_{i}$ and $u_{i}$. They are covered by the following lemmas.

Lemma 3. Assume (21). Then there exists a positive constant $C$ such that

$$
\sum_{i=1}^{n}\left\|\nabla \phi_{c_{i}}\right\|_{\mathbf{L}^{2}(T)}^{2} \tau \leqslant C \text {. }
$$

Proof. Take $\xi=\phi_{c_{i}} \tau$ in (25) and sum it up for $i=1, \ldots, l \leqslant n$ to get

$$
\sum_{i=1}^{l}\left(\sigma_{\pi}\left(u_{i-1}\right) \nabla \phi_{c_{i}}, \nabla \phi_{c_{i}}\right)_{T} \tau=-\sum_{i=1}^{l}\left(j_{i}, \phi_{c_{i}}\right)_{\Gamma} \tau .
$$

We bound the left-hand side (LHS) from below

$$
\sigma_{*} \sum_{i=1}^{l}\left\|\nabla \phi_{c_{i}}\right\|_{\mathbf{L}^{2}(T)}^{2} \tau \leqslant \sum_{i=1}^{l}\left(\sigma_{\pi}\left(u_{i-1}\right) \nabla \phi_{c_{i}}, \nabla \phi_{c_{i}}\right)_{T} \tau .
$$

Using Cauchy-Schwarz's and Young's inequalities, we bound the RHS

$$
\sum_{i=1}^{l}\left(j_{i}, \phi_{c_{i}}\right)_{\Gamma} \tau \leqslant \frac{1}{2 \varepsilon} \sum_{i=1}^{l}\left\|j_{i}\right\|_{H^{-1 / 2}(\Gamma)}^{2} \tau+\frac{\varepsilon}{2} \sum_{i=1}^{l}\left\|\phi_{c_{i}}\right\|_{H^{1 / 2}(\Gamma)}^{2} \tau \leqslant C_{\varepsilon}+\varepsilon \sum_{i=1}^{l}\left\|\phi_{c_{i}}\right\|_{H^{1 / 2}(\Gamma)}^{2} \tau,
$$


where $\varepsilon>0$. Since $H^{1}(T) / \mathbb{R} \subset H^{1 / 2}(\Gamma)$ we use Lemma 1 to write

$$
\sum_{i=1}^{l}\left\|\phi_{c_{i}}\right\|_{H^{1 / 2}(\Gamma)}^{2} \tau \leqslant C \sum_{i=1}^{l}\left\|\nabla \phi_{c_{i}}\right\|_{\mathbf{L}^{2}(T)}^{2} \tau .
$$

Now fixing a sufficiently small $\varepsilon$ we conclude the proof.

Lemma 4. Assume (21). Then there exists a positive constant $C$ such that

(i) $\sum_{i=1}^{n}\left\|\delta \boldsymbol{A}_{i}\right\|_{\mathbf{L}^{2}(\pi)}^{2} \tau+\max _{1 \leqslant l \leqslant n}\left\|\nabla \times \boldsymbol{A}_{l}\right\|_{\mathbf{L}^{2}(\Omega)}^{2} \leqslant C$

(ii) $\sum_{i=1}^{n}\left\|\nabla \times\left(\mu \boldsymbol{M}\left(\nabla \times \boldsymbol{A}_{i}\right)\right)\right\|_{\mathbf{L}^{2}(\pi)}^{2} \tau \leqslant C$.

Proof. (i) Taking $\boldsymbol{\varphi}=\delta \boldsymbol{A}_{i} \tau$ in (26) and summing up for $i=1, \ldots, l \leqslant n$ yields

$$
\sum_{i=1}^{l}\left(\sigma_{\pi}\left(u_{i-1}\right) \delta \boldsymbol{A}_{i}, \delta \boldsymbol{A}_{i}\right)_{\pi} \tau+\sum_{i=1}^{l}\left(\mu \boldsymbol{M}\left(\nabla \times \boldsymbol{A}_{i}\right), \nabla \times \boldsymbol{A}_{i}-\nabla \times \boldsymbol{A}_{i-1}\right)_{\Omega}=-\sum_{i=1}^{l}\left(\sigma_{\pi}\left(u_{i-1}\right) \nabla \phi_{c_{i}}, \delta \boldsymbol{A}_{i}\right)_{T} \tau .
$$

Using Lemma 3, Cauchy-Schwarz's and Young's inequalities, we bound the first term on the LHS and the term on the RHS as follows

$$
\begin{aligned}
\sigma_{*} \sum_{i=1}^{l}\left\|\delta \boldsymbol{A}_{i}\right\|_{\mathbf{L}^{2}(\pi)}^{2} \tau & \leqslant \sum_{i=1}^{l}\left(\sigma_{\pi}\left(u_{i-1}\right) \delta \boldsymbol{A}_{i}, \delta \boldsymbol{A}_{i}\right)_{\pi} \tau \\
-\sum_{i=1}^{l}\left(\sigma_{\pi}\left(u_{i-1}\right) \nabla \phi_{c_{i}}, \delta \boldsymbol{A}_{i}\right)_{T} \tau & \leqslant \frac{\sigma^{*}}{2 \varepsilon} \sum_{i=1}^{l}\left\|\nabla \phi_{c_{i}}\right\|_{\mathbf{L}^{2}(T)}^{2} \tau+\frac{\varepsilon \sigma^{*} C_{\pi}}{2} \sum_{i=1}^{l}\left\|\delta \boldsymbol{A}_{i}\right\|_{\mathbf{L}^{2}(\pi)}^{2} \tau . \\
& \leqslant C \frac{\sigma^{*}}{2 \varepsilon}+\frac{\varepsilon \sigma^{*} C_{\pi}}{2} \sum_{i=1}^{l}\left\|\delta \boldsymbol{A}_{i}\right\|_{\mathbf{L}^{2}(\pi)}^{2} \tau .
\end{aligned}
$$

To estimate the second term on the LHS, we take into account (23) and (24)

$$
\begin{gathered}
\sum_{i=1}^{l} \int_{\Omega} \mu\left\{\boldsymbol{M}\left(\nabla \times \boldsymbol{A}_{i}\right) \cdot\left(\nabla \times \boldsymbol{A}_{i}-\nabla \times \boldsymbol{A}_{i-1}\right)\right\} \mathrm{d} x \\
\geqslant \sum_{i=1}^{l} \int_{\Omega} \mu\left(\Phi_{\boldsymbol{M}}\left(\nabla \times \boldsymbol{A}_{i}\right)-\Phi_{\boldsymbol{M}}\left(\nabla \times \boldsymbol{A}_{i-1}\right)\right) \mathrm{d} x \\
\quad=\int_{\Omega} \mu \Phi_{\boldsymbol{M}}\left(\nabla \times \boldsymbol{A}_{l}\right) \mathrm{d} x-\int_{\Omega} \mu \Phi_{\boldsymbol{M}}\left(\nabla \times \boldsymbol{A}_{0}\right) \mathrm{d} x \\
\geqslant \frac{c_{M} \mu_{*}}{2}\left\|\nabla \times \boldsymbol{A}_{l}\right\|_{\mathbf{L}^{2}(\Omega)}^{2}-\frac{C_{M} \mu^{*}}{2}\left\|\nabla \times \boldsymbol{A}_{0}\right\|_{\mathbf{L}^{2}(\Omega)}^{2} .
\end{gathered}
$$

We relocate the terms to get

$$
\left(\sigma_{*}-\frac{\varepsilon}{2} \sigma^{*} C_{\pi}\right) \sum_{i=1}^{l}\left\|\delta \boldsymbol{A}_{i}\right\|_{\mathbf{L}^{2}(\pi)}^{2} \tau+\frac{c_{M} \mu_{*}}{2}\left\|\nabla \times \boldsymbol{A}_{l}\right\|_{\mathbf{L}^{2}(\Omega)}^{2} \leqslant C \frac{\sigma^{*}}{2 \varepsilon}+\frac{C_{M} \mu^{*}}{2}\left\|\nabla \times \boldsymbol{A}_{0}\right\|_{\mathbf{L}^{2}(\Omega)}^{2} .
$$

Fixing $\varepsilon \in\left(0, \frac{2 \sigma_{*}}{\sigma^{*} C_{\pi}}\right)$ and assuming that $\boldsymbol{A}_{0} \in \boldsymbol{X}_{N, 0}$, we obtain

$$
\sum_{i=1}^{l}\left\|\delta \boldsymbol{A}_{i}\right\|_{\mathbf{L}^{2}(\pi)}^{2} \tau+\left\|\nabla \times \boldsymbol{A}_{l}\right\|_{\mathbf{L}^{2}(\Omega)}^{2} \leqslant C .
$$

This is valid for any $1 \leqslant l \leqslant n$, which concludes the proof of (i).

(ii) Take $\boldsymbol{\varphi} \in \mathbf{C}_{0}^{\infty}(\boldsymbol{\pi})$. It holds

$$
\begin{gathered}
\left(\sigma_{\pi}\left(u_{i-1}\right) \delta \boldsymbol{A}_{i}, \boldsymbol{\varphi}\right)_{\pi}+\left(\sigma_{\pi}\left(u_{i-1}\right) \nabla \boldsymbol{\phi}_{c_{i}}, \boldsymbol{\varphi}\right)_{T}=-\left(\mu \boldsymbol{M}\left(\nabla \times \boldsymbol{A}_{i}\right), \nabla \times \boldsymbol{\varphi}\right)_{\Omega} \\
\stackrel{\text { Green's theorem }}{=}-\left(\nabla \times\left(\mu \boldsymbol{M}\left(\nabla \times \boldsymbol{A}_{i}\right)\right), \boldsymbol{\varphi}\right)_{\Omega} .
\end{gathered}
$$


Based on Lemmas 3 and 4(i) we see that the LHS can be seen as a linear bounded functional in $L^{2}\left((0, \mathcal{T}) ; \mathbf{L}^{2}(\boldsymbol{\pi})\right)$. According to the Hahn-Banach theorem the same holds true for the RHS, i.e.

$$
\sum_{i=1}^{n}\left\|\nabla \times\left(\mu \boldsymbol{M}\left(\nabla \times \boldsymbol{A}_{i}\right)\right)\right\|_{\mathbf{L}^{2}(\pi)}^{2} \tau \leqslant C .
$$

Lemma 5. Let (21) be fulfilled. Then there exists a positive constant $C_{r}$, depending only on parameter $r$ of truncation function $\mathcal{R}_{r}$, such that

$$
\begin{aligned}
& \text { (i) } \sum_{i=1}^{n}\left\|\delta u_{i}\right\|_{L^{2}(\pi)}^{2} \tau+\sum_{i=1}^{n}\left\|\nabla u_{i}-\nabla u_{i-1}\right\|_{\mathbf{L}^{2}(\pi)}^{2}+\max _{1 \leqslant i \leqslant n}\left\|\nabla u_{i}\right\|_{\mathbf{L}^{2}(\pi)} \leqslant C_{r}, \\
& \text { (ii) } \max _{1 \leqslant i \leqslant n}\left\|u_{i}\right\|_{L^{2}(\pi)}^{2} \leqslant C_{r}, \\
& \text { (iii) } \max _{1 \leqslant i \leqslant n}\left\|\delta \beta\left(u_{i}\right)\right\|_{\left(H^{1}(\pi)\right)^{*}}^{2} \leqslant C_{r} .
\end{aligned}
$$

Proof. (i) Take $\psi=\delta u_{i} \tau$ in (27) and sum it up for $i=1, \ldots, l \leqslant n$ to have

$$
\begin{aligned}
& \sum_{i=1}^{l}\left(\delta \beta\left(u_{i}\right), \delta u_{i}\right)_{\pi} \tau+\sum_{i=1}^{l}\left(\lambda_{i} \nabla u_{i}, \nabla u_{i}-\nabla u_{i-1}\right)_{\pi} \\
& =\sum_{i=1}^{l}\left(\mathcal{R}_{r}\left(\sigma_{\pi}\left(u_{i-1}\right)\left|\delta \boldsymbol{A}_{i}+\chi_{T} \nabla \phi_{c_{i}}\right|^{2}\right), \delta u_{i}\right)_{\pi} \tau .
\end{aligned}
$$

Utilizing the mean value theorem and (21), we bound the first term on the LHS

$$
\sum_{i=1}^{l}\left(\delta \beta\left(u_{i}\right), \delta u_{i}\right)_{\pi} \tau=\sum_{i=1}^{l}\left(\beta^{\prime}(\eta)\left(u_{i}-u_{i-1}\right), \delta u_{i}\right)_{\pi} \geqslant \beta_{*} \sum_{i=1}^{l}\left\|\delta u_{i}\right\|_{L^{2}(\pi)}^{2} \tau .
$$

For the term on the RHS we use Cauchy's and Young's inequalities

$$
\begin{aligned}
\sum_{i=1}^{l}\left(\mathcal{R}_{r}\left(\sigma_{\pi}\left(u_{i-1}\right)\left|\delta \boldsymbol{A}_{i}+\chi_{T} \nabla \phi_{c_{i}}\right|^{2}\right), \delta u_{i}\right)_{\pi} \tau & \leqslant \frac{C_{r}^{2}}{2 \varepsilon}|\boldsymbol{\pi}| \mathcal{T}+\frac{\varepsilon}{2} \sum_{i=1}^{l}\left\|\delta u_{i}\right\|_{L^{2}(\pi)}^{2} \tau \\
& =C_{r, \varepsilon}+\frac{\varepsilon}{2} \sum_{i=1}^{l}\left\|\delta u_{i}\right\|_{L^{2}(\pi)}^{2} \tau .
\end{aligned}
$$

Thanks to Lipschitz continuity of $\lambda$ in time, we bound the last term as follows (cf. [26])

$$
\begin{aligned}
\sum_{i=1}^{l}\left(\lambda_{i} \nabla u_{i}, \nabla u_{i}-\nabla u_{i-1}\right)_{\pi}= & \frac{1}{2} \int_{\pi} \lambda_{l}\left|\nabla u_{l}\right|^{2} \mathrm{~d} x+\frac{1}{2} \sum_{i=1}^{l} \int_{\pi} \lambda_{i}\left|\nabla u_{i}-\nabla u_{i-1}\right|^{2} \mathrm{~d} x \\
& -\frac{1}{2} \int_{\pi} \lambda_{1}\left|\nabla u_{0}\right|^{2} \mathrm{~d} x-\frac{1}{2} \sum_{i=1}^{l} \int_{\pi}\left(\lambda_{i+1}-\lambda_{i}\right)\left|\nabla u_{i}\right|^{2} \mathrm{~d} x \\
\geqslant & \frac{\lambda_{*}}{2}\left\|\nabla u_{l}\right\|_{\mathbf{L}^{2}(\pi)}^{2}+\frac{\lambda_{*}}{2} \sum_{i=1}^{l}\left\|\nabla u_{i}-\nabla u_{i-1}\right\|_{\mathbf{L}^{2}(\pi)}^{2} \\
& -\frac{C_{\lambda}}{2} \sum_{i=0}^{l-1}\left\|\nabla u_{i}\right\|_{\mathbf{L}^{2}(\pi)}^{2} \tau-\frac{\lambda^{*}}{2}\left\|\nabla u_{0}\right\|_{\mathbf{L}^{2}(\pi)}^{2} .
\end{aligned}
$$

Collecting all estimates above, taking $\varepsilon \in\left(0,2 \beta_{*}\right)$ and using Grönwall's lemma we obtain (i).

(ii) This part follows readily from (i) and

$$
u_{l}=u_{0}+\sum_{i=1}^{l} \delta u_{i} \tau \Longrightarrow\left\|u_{l}\right\|_{L^{2}(\pi)} \leqslant\left\|u_{0}\right\|_{L^{2}(\pi)}+\sum_{i=1}^{l}\left\|\delta u_{i}\right\|_{L^{2}(\pi)} \tau \leqslant C_{r}
$$

for any $0 \leqslant l \leqslant n$. 
(iii) Norm in $\left(H^{1}(\pi)\right)^{*}$ is defined as

$$
\|u\|_{\left(H^{1}(\pi)\right)^{*}}:=\sup _{\psi \neq 0} \frac{\left|(u, \psi)_{\pi}\right|}{\|\psi\|_{H^{1}(\pi)}} .
$$

Thus, deducing from (27) and using estimates above we write

$$
\begin{aligned}
\left|\left(\delta \beta\left(u_{i}\right), \psi\right)_{\pi}\right| & \leqslant\left|\left(\mathcal{R}_{r}\left(\sigma_{\pi}\left(u_{i-1}\right)\left|\delta \boldsymbol{A}_{i}+\chi_{T} \nabla \phi_{c_{i}}\right|^{2}\right), \psi\right)_{\pi}\right|+\left|\left(\nabla u_{i}, \nabla \psi\right)_{\pi}\right| \\
& \leqslant C_{r} \sqrt{|\boldsymbol{\pi}|}\|\psi\|_{L^{2}(\pi)}+\left\|\nabla u_{i}\right\|_{\mathbf{L}^{2}(\pi)}\|\nabla \psi\|_{\mathbf{L}^{2}(\pi)} \\
& \leqslant\left\{C_{r} \sqrt{|\boldsymbol{\pi}|}+\left\|\nabla u_{i}\right\|_{\mathbf{L}^{2}(\pi)}\right\}\|\psi\|_{H^{1}(\pi)} \\
& \leqslant C_{r}\|\psi\|_{H^{1}(\pi)},
\end{aligned}
$$

therefore

$$
\left\|\delta \beta\left(u_{i}\right)\right\|_{\left(H^{1}(\pi)\right)^{*}} \leqslant C_{r},
$$

for any $i=1, \ldots, n$.

\subsection{Convergence}

The existence of a weak solution-triplet $\{\boldsymbol{A}, \phi, u\}$ of (18)-(20) is shown in this section. We construct Rothe's functions and prove that they converge towards a weak solution of our system. Before we state the main theorem where the existence of a weak solution is proven we introduce 2 propositions.

In the first proposition we use well known results from the functional analysis valid in parabolic partial differential equations containing Gelfand's triple, cf. [23,27]. In this manner we obtain the convergence results for the approximate solution of temperature.

In the second proposition we use the monotone character of vector field $\boldsymbol{M}$ and the technique of Minty-Browder, cf. $[28,29]$ to overcome the nonlinearity when passing to the limit.

We start by introducing Rothe's functions. They are piece-wise constant and piece-wise linear in time functions and are constructed in the following way for $i=1, \ldots, n$ (where $n$ denotes the number of time steps)

$$
\begin{array}{llll}
\overline{\phi_{n}}(t)=\phi_{c_{i}} & & \text { for } t \in\left(t_{i-1}, t_{i}\right], & \\
\overline{\boldsymbol{A}_{n}}(t)=\boldsymbol{A}_{i} & \boldsymbol{A}_{n}(t)=\boldsymbol{A}_{i-1}+\left(t-t_{i-1}\right) \delta \boldsymbol{A}_{i} & \text { for } t \in\left(t_{i-1}, t_{i}\right] & \overline{\boldsymbol{A}_{n}}(0)=\boldsymbol{A}_{n}(0)=\boldsymbol{A}_{0}, \\
\overline{u_{n}}(t)=u_{i} & u_{n}(t)=u_{i-1}+\left(t-t_{i-1}\right) \delta u_{i} & \text { for } t \in\left(t_{i-1}, t_{i}\right] & \overline{u_{n}}(0)=u_{n}(0)=u_{0}, \\
\overline{\beta_{n}}(t)=\beta\left(u_{i}\right) & \beta_{n}(t)=\beta\left(u_{i-1}\right)+\left(t-t_{i-1}\right) \delta \beta\left(u_{i}\right) & \text { for } t \in\left(t_{i-1}, t_{i}\right] & \overline{\beta_{n}}(0)=\beta_{n}(0)=\beta\left(u_{0}\right), \\
\overline{j_{n}}(t)=j_{i} & \overline{\lambda_{n}}(t)=\lambda_{i} \quad \overline{\sigma_{\pi n}}(t)=\sigma_{\pi}\left(u_{i}\right) & \text { for } t \in\left(t_{i-1}, t_{i}\right] . &
\end{array}
$$

For better interpretation we include a simple example of Rothe's functions for a general function $f(t)$ in Fig. 3 . Now, using these new notations we rewrite $(25)-(27)$ in a continuous sense for the whole time interval $[0, \mathcal{T}]$, i.e.

$$
\begin{aligned}
\left(\overline{\sigma_{\pi_{n}}}(t-\tau) \nabla \overline{\phi_{n}}, \nabla \xi\right)_{T}+\left(\overline{j_{n}}, \xi\right)_{\Gamma}=0 & \text { for any } \xi \in H^{1}(T) / \mathbb{R}, \\
\left(\overline{\sigma_{\pi_{n}}}(t-\tau) \partial_{t} \boldsymbol{A}_{n}, \boldsymbol{\varphi}\right)_{\pi}+\left(\mu \boldsymbol{M}\left(\nabla \times \overline{\boldsymbol{A}_{n}}\right), \nabla \times \varphi\right)_{\Omega}+\left(\overline{\sigma_{\pi_{n}}}(t-\tau) \nabla \overline{\phi_{n}}, \boldsymbol{\varphi}\right)_{T}=0 & \text { for any } \boldsymbol{\varphi} \in \boldsymbol{X}_{N, 0}, \\
\left(\partial_{t} \beta_{n}, \psi\right)_{\pi}+\left(\overline{\lambda_{n}} \nabla \overline{u_{n}}, \psi\right)_{\pi}-\left(\mathcal{R}_{r}\left(\overline{\sigma_{\pi_{n}}}(t-\tau)\left|\partial_{t} \boldsymbol{A}_{n}+\chi_{T} \nabla \overline{\phi_{n}}\right|^{2}\right), \psi\right)_{\pi}=0 & \text { for any } \psi \in H^{1}(\boldsymbol{\pi}) .
\end{aligned}
$$

Proposition 1. Suppose (21). Moreover assume that $\sigma$ is globally Lipschitz continuous. Then there exist a scalar function $u \in C\left([0, \mathcal{T}] ; L^{2}(\pi)\right) \cap L^{\infty}\left((0, \mathcal{T}) ; H_{0}^{1}(\pi)\right)$ with $\partial_{t} u \in L^{2}\left((0, \mathcal{T}) ; L^{2}(\pi)\right)$ and a sub-sequence of $u_{n}($ denoted 


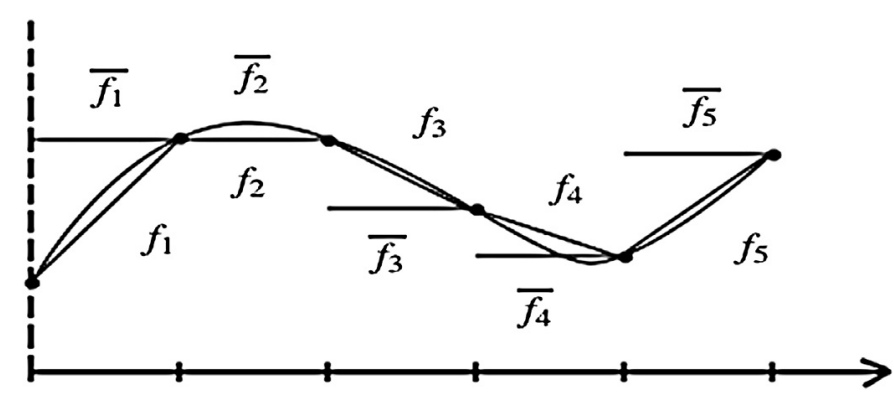

Fig. 3. Rothe's functions of a general function $f(t)$.

by the same symbol again) such that
(i) $u_{n} \rightarrow u$
$\overline{u_{n}}(t) \rightarrow u(t)$
$\overline{u_{n}} \rightarrow u$
in $C\left([0, \mathcal{T}] ; L^{2}(\pi)\right)$, in $H^{1}(\pi), \forall t \in[0, \mathcal{T}]$,
(ii) $\quad \overline{\sigma_{\pi_{n}}} \rightarrow \sigma_{\pi}(u), \overline{\sigma_{\pi_{n}}}(t-\tau) \rightarrow \sigma_{\pi}(u)$ in $L^{2}\left((0, \mathcal{T}) ; L^{2}(\pi)\right)$,
(iii) $\quad \overline{\beta_{n}}-\beta_{n} \rightarrow 0$
in $L^{2}\left((0, \mathcal{T}) ; L^{2}(\pi)\right)$,
(iv) $\overline{\beta_{n}} \rightarrow \beta(u)$
in $C\left([0, \mathcal{T}] ;\left(H^{1}(\pi)\right)^{*}\right)$,
(v) $\overline{j_{n}} \rightarrow j$
in $L^{2}\left((0, \mathcal{T}) ; L^{2}(\pi)\right)$,
in $L^{2}\left((0, \mathcal{T}) ; H^{-1 / 2}(\Gamma)\right)$

holds true for $n \rightarrow+\infty$.

Proof. (i) Using Lemma 5, we have $\partial_{t} u_{n} \in L^{2}\left((0, \mathcal{T}) ; L^{2}(\pi)\right)$ and $\overline{u_{n}} \in C\left([0, \mathcal{T}] ; H^{1}(\pi)\right)$. Now, since $H^{1}(\pi)$ is compactly embedded in $L^{2}(\pi)$, we apply well-known [23, Lemma 1.3.13] to conclude the first two statements of (i). To prove the last one we only need to show that $u_{n}$ and $\overline{u_{n}}$ have the same limit in $L^{2}\left((0, \mathcal{T}) ; L^{2}(\pi)\right)$. We may write

$$
\begin{aligned}
\int_{0}^{\mathcal{T}}\left\|\overline{u_{n}}-u_{n}\right\|_{L^{2}(\pi)}^{2} \mathrm{~d} t & =\sum_{i=1}^{n} \int_{t_{i-1}}^{t_{i}}\left\|u_{i}-u_{i-1}-\left(t-t_{i-1}\right) \delta u_{i}\right\|_{L^{2}(\pi)}^{2} \mathrm{~d} t \\
& =\sum_{i=1}^{n} \int_{t_{i-1}}^{t_{i}}\left\|\delta u_{i}\left(\tau-t+t_{i-1}\right)\right\|_{L^{2}(\pi)}^{2} \mathrm{~d} t \\
& \leqslant \tau^{2} \sum_{i=1}^{n}\left\|\delta u_{i}\right\|_{L^{2}(\pi)}^{2} \tau \leqslant C_{r} \tau^{2} \stackrel{n \rightarrow \infty}{\longrightarrow} 0 .
\end{aligned}
$$

(ii) Since $\sigma$ is supposed to be globally Lipschitz continuous and $\overline{u_{n}}$ converges strongly to $u$ in $L^{2}\left((0, \mathcal{T}) ; L^{2}(\pi)\right)$, we conclude that $\overline{\sigma_{\pi_{n}}} \rightarrow \sigma(u)$ in the same space as well. The only thing left to be done is to show that $\overline{\sigma_{\pi_{n}}}(t-\tau)$ and $\overline{\sigma_{\pi_{n}}}(t)$ share the same limit in $L^{2}\left((0, \mathcal{T}) ; L^{2}(\pi)\right)$. It holds

$$
\begin{aligned}
& \int_{0}^{\mathcal{T}}\left\|\overline{\sigma_{\pi_{n}}}(t)-\overline{\sigma_{\pi_{n}}}(t-\tau)\right\|_{L^{2}(\pi)}^{2} \mathrm{~d} t=\sum_{i=1}^{n}\left\|\sigma\left(u_{i}\right)-\sigma\left(u_{i-1}\right)\right\|_{L^{2}(\pi)}^{2} \tau \\
& \stackrel{\text { Lipschitz }}{\leqslant} C_{\sigma} \sum_{i=1}^{n}\left\|u_{i}-u_{i-1}\right\|_{L^{2}(\pi)}^{2} \tau \\
&=C_{\sigma} \tau^{2} \sum_{i=1}^{n}\left\|\delta u_{i}\right\|_{L^{2}(\pi)}^{2} \tau \leqslant C_{\sigma} C_{r} \tau^{2} \stackrel{n \rightarrow \infty}{\longrightarrow} 0 .
\end{aligned}
$$

(iii) Results from Lemma 5 let us write

$$
\left|\left(\overline{\beta_{n}}-\beta_{n}, \psi\right)\right| \leqslant \tau\left\|\partial_{t} \beta_{n}\right\|_{\left(H^{1}(\pi)\right)^{*}}\|\psi\|_{H^{1}(\pi)} \leqslant \tau C_{r}\|\psi\|_{H^{1}(\pi)}
$$

and therefore $\left\|\overline{\beta_{n}}-\beta_{n}\right\|_{\left(H^{1}(\pi)\right)^{*}} \leqslant \tau C_{r} \stackrel{n \rightarrow \infty}{\longrightarrow} 0$. 
(iv) Taking into account the continuity of $\beta$ and the fact that $u_{n}$ converges strongly towards $u$, allow us to use Lebesgue's dominated convergence theorem to conclude that $\overline{\beta_{n}} \rightarrow \beta(u)$ in $L^{2}\left((0, \mathcal{T}) ; L^{2}(\pi)\right)$.

(v) Assuming that $j$ is Lipschitz continuous in time, we write

$$
\int_{0}^{\mathcal{T}}\left\|\overline{j_{n}}-j\right\|_{H^{-1 / 2}(\Gamma)}^{2} \mathrm{~d} t=\sum_{i=1}^{n} \int_{t_{i-1}}^{t_{i}}\left\|j\left(t_{i}\right)-j(t)\right\|_{H^{-1 / 2}(\Gamma)}^{2} \mathrm{~d} t \leqslant C \tau^{2} \stackrel{n \rightarrow \infty}{\rightarrow} 0 .
$$

Proposition 2. Suppose that all assumptions of Proposition 1 are satisfied. Then there exist a vector potential $\boldsymbol{A} \in L^{2}\left((0, \mathcal{T}) ; X_{N, 0}\right)$ with $\partial_{t} \boldsymbol{A} \in L^{2}\left((0, \mathcal{T}) ; \mathbf{L}^{2}(\pi)\right)$ and a sub-sequence of $\boldsymbol{A}_{n}$ (denoted by the same symbol again) such that
(i) $\overline{\boldsymbol{A}_{n}} \rightarrow \boldsymbol{A}, \nabla \times \overline{\boldsymbol{A}_{n}} \rightarrow \nabla \times \boldsymbol{A}$ $\mu \boldsymbol{M}\left(\nabla \times \overline{A_{n}}\right) \rightarrow \mu \boldsymbol{M}(\nabla \times \boldsymbol{A})$
$\boldsymbol{A}_{n} \rightarrow \boldsymbol{A}$$$
\boldsymbol{A}_{n}(t) \rightarrow \boldsymbol{A}(t), \overline{\boldsymbol{A}_{n}}(t) \rightarrow \boldsymbol{A}(t)
$$$$
\partial_{t} \boldsymbol{A}_{n} \rightarrow \partial_{t} \boldsymbol{A}
$$
in $\quad L^{2}\left((0, \mathcal{T}) ; \mathbf{L}^{2}(\Omega)\right)$,
in $L^{2}\left((0, \mathcal{T}) ; \mathbf{L}^{2}(\Omega \backslash \boldsymbol{\pi})\right)$,
in $C\left([0, \mathcal{T}] ; \mathbf{L}^{2}(\boldsymbol{\pi})\right)$,
in $\mathbf{H}^{1}(\boldsymbol{\pi}), \forall t$,
in $L^{2}\left((0, \mathcal{T}) ; \mathbf{L}^{2}(\boldsymbol{\pi})\right)$,
$\partial_{t} A_{n} \rightarrow \partial_{t} A$
(ii) $\boldsymbol{M}\left(\nabla \times \overline{A_{n}}\right) \rightarrow \boldsymbol{M}(\nabla \times \boldsymbol{A})$
in $\quad L^{2}\left((0, \mathcal{T}) ; \mathbf{L}^{2}(\boldsymbol{\pi})\right)$,
(iii) $\nabla \times \overline{\boldsymbol{A}_{n}} \rightarrow \nabla \times \boldsymbol{A}$
$\boldsymbol{M}\left(\nabla \times \overline{A_{n}}\right) \rightarrow \boldsymbol{M}(\nabla \times \boldsymbol{A})$
in $\quad L^{2}\left((0, \mathcal{T}) ; \mathbf{L}^{2}(\boldsymbol{\pi})\right)$,
in $L^{2}\left((0, \mathcal{T}) ; \mathbf{L}^{2}(\boldsymbol{\pi})\right)$

holds true for $n \rightarrow+\infty$.

Proof. (i) Lemma 4 yields

$$
\int_{0}^{\mathcal{T}}\left\|\overline{\boldsymbol{A}_{n}}\right\|_{\boldsymbol{X}_{N, 0}}^{2} \mathrm{~d} t \leqslant C
$$

The reflexivity of $L^{2}\left((0, \mathcal{T}) ; \boldsymbol{X}_{N, 0}\right)$ gives for a sub-sequence that $\overline{\boldsymbol{A}_{n}} \rightarrow \boldsymbol{A}$ in that space. One can easily see that

$$
\overline{\boldsymbol{A}_{n}} \rightarrow \boldsymbol{A}, \quad \nabla \times \overline{\boldsymbol{A}_{n}} \rightarrow \nabla \times \boldsymbol{A} \quad \text { in } L^{2}\left((0, \mathcal{T}) ; \mathbf{L}^{2}(\Omega)\right),
$$

due to the density of $\boldsymbol{C}_{0}^{\infty}(\Omega)$ in $\mathbf{L}^{2}(\Omega)$, see [30, Thm. 2.6.1]. Take now $\boldsymbol{\varphi} \in \mathbf{C}_{0}^{\infty}(\Omega \backslash \boldsymbol{\pi})$. Using $\mu \in H^{1}(\Omega \backslash \boldsymbol{\pi})$ we have

$$
\int_{0}^{\mathcal{T}}\left(\mu \boldsymbol{M}\left(\nabla \times \overline{\boldsymbol{A}_{n}}\right), \varphi\right)_{\Omega} \mathrm{d} t=\int_{0}^{\mathcal{T}}\left(\mu \nabla \times \overline{\boldsymbol{A}_{n}}, \boldsymbol{\varphi}\right)_{\Omega} \mathrm{d} t=\int_{0}^{\mathcal{T}}\left(\overline{\boldsymbol{A}_{n}}, \nabla \times(\mu \varphi)\right)_{\Omega} \mathrm{d} t .
$$

Passing to the limit for $n \rightarrow \infty$ we get

$$
\lim _{n \rightarrow \infty} \int_{0}^{\mathcal{T}}\left(\mu \nabla \times \overline{\boldsymbol{A}_{n}}, \boldsymbol{\varphi}\right)_{\Omega} \mathrm{d} t=\int_{0}^{\mathcal{T}}(\boldsymbol{A}, \nabla \times(\mu \boldsymbol{\varphi}))_{\Omega} \mathrm{d} t=\int_{0}^{\mathcal{T}}(\mu \nabla \times \boldsymbol{A}, \boldsymbol{\varphi})_{\Omega} \mathrm{d} t .
$$

Using the density argument of $\mathbf{C}_{0}^{\infty}(\Omega \backslash \boldsymbol{\pi})$ in $\mathbf{L}^{2}(\Omega \backslash \boldsymbol{\pi})$ we have

$$
\mu \boldsymbol{M}\left(\nabla \times \overline{\boldsymbol{A}_{n}}\right)=\mu \nabla \times \overline{\boldsymbol{A}_{n}} \rightarrow \mu \nabla \times \boldsymbol{A}=\mu \boldsymbol{M}(\nabla \times \boldsymbol{A}) \quad \text { in } L^{2}\left((0, \mathcal{T}) ; \mathbf{L}^{2}(\Omega \backslash \boldsymbol{\pi})\right) .
$$

Lemma 4 together with $\boldsymbol{X}_{N, 0} \subset \mathbf{H}^{1}(\Omega)$ (cf. [19, Theorem 3.7]) imply

$$
\int_{0}^{\mathcal{T}}\left\|\partial_{t} \boldsymbol{A}_{n}\right\|_{L^{2}(\pi)}^{2} \mathrm{~d} t \leqslant C, \quad\left\|\overline{\boldsymbol{A}_{n}}\right\|_{\mathbf{H}^{1}(\pi)} \leqslant\left\|\overline{\boldsymbol{A}_{n}}\right\|_{\mathbf{H}^{1}(\Omega)} \leqslant C .
$$

Employing [23, Lemma 1.3.13] we get for a sub-sequence that

$$
\begin{array}{ll}
\boldsymbol{A}_{n} \rightarrow \boldsymbol{A} & \text { in } \quad C\left([0, \mathcal{T}] ; \mathbf{L}^{2}(\boldsymbol{\pi})\right) \\
\boldsymbol{A}_{n}(t) \rightarrow \boldsymbol{A}(t), \overline{\boldsymbol{A}_{n}}(t) \rightarrow \boldsymbol{A}(t) & \text { in } \mathbf{H}^{1}(\boldsymbol{\pi}), \forall t \\
\partial_{t} \boldsymbol{A}_{n} \rightarrow \partial_{t} \boldsymbol{A} & \text { in } L^{2}\left((0, \mathcal{T}) ; \mathbf{L}^{2}(\boldsymbol{\pi})\right) .
\end{array}
$$

(ii) The sequence $\boldsymbol{M}\left(\nabla \times \overline{\boldsymbol{A}_{n}}\right)$ is bounded in $L^{2}\left((0, \mathcal{T}) ; \mathbf{L}^{2}(\Omega)\right)$. Therefore, there exists $\boldsymbol{p}$ from $L^{2}\left((0, \mathcal{T}) ; \mathbf{L}^{2}(\Omega)\right)$ such that $\boldsymbol{M}\left(\nabla \times \overline{\boldsymbol{A}_{n}}\right) \rightarrow \boldsymbol{p}$ in that space (for a sub-sequence). Now, we involve the remarkable Minty-Browder 
technique, cf. [31,18]. The general idea is based on monotone character of the vectorial field $\boldsymbol{M}$. Let us investigate the following inequality

$$
0 \leqslant \int_{0}^{\mathcal{T}}\left(\boldsymbol{M}\left(\nabla \times \overline{\boldsymbol{A}_{n}}\right)-\boldsymbol{M}(\boldsymbol{b}), \psi \mu\left(\nabla \times \overline{\boldsymbol{A}_{n}}-\boldsymbol{b}\right)\right)_{\Omega} \mathrm{d} t=I_{1}+I_{2}+I_{3}+I_{4},
$$

where

$$
\begin{array}{rlrl}
I_{1} & =\int_{0}^{\mathcal{T}}\left(\boldsymbol{M}\left(\nabla \times \overline{\boldsymbol{A}_{n}}\right), \psi \mu \nabla \times \overline{\boldsymbol{A}_{n}}\right)_{\Omega} \mathrm{d} t, & I_{2}=\int_{0}^{\mathcal{T}}\left(\boldsymbol{M}(\boldsymbol{b}), \psi \mu \nabla \times \overline{\boldsymbol{A}_{n}}\right)_{\Omega} \mathrm{d} t, \\
I_{3}=\int_{0}^{\mathcal{T}}\left(\boldsymbol{M}\left(\nabla \times \overline{\boldsymbol{A}_{n}}\right), \psi \mu \boldsymbol{b}\right)_{\Omega} \mathrm{d} t, & I_{4}=\int_{0}^{\mathcal{T}}(\boldsymbol{M}(\boldsymbol{b}), \psi \mu \boldsymbol{b})_{\Omega} \mathrm{d} t .
\end{array}
$$

This inequality holds true for any $\boldsymbol{b} \in L^{2}\left((0, \mathcal{T}) ; \mathbf{L}^{2}(\Omega)\right)$ and any non-negative $\psi \in C_{0}^{\infty}(\boldsymbol{\pi})$. We want to pass to the limit for $n \rightarrow \infty$ in (32). We do it for each term in (32) separately.

It holds

$$
\begin{aligned}
I_{1}= & \int_{0}^{\mathcal{T}}\left(\boldsymbol{M}\left(\nabla \times \overline{\boldsymbol{A}_{n}}\right), \psi \mu \nabla \times \overline{\boldsymbol{A}_{n}}\right)_{\Omega} \mathrm{d} t \\
= & \int_{0}^{\mathcal{T}}\left(\boldsymbol{M}\left(\nabla \times \overline{\boldsymbol{A}_{n}}\right), \psi \mu \nabla \times\left(\overline{\boldsymbol{A}_{n}}-\boldsymbol{A}\right)\right)_{\Omega} \mathrm{d} t+\int_{0}^{\mathcal{T}}\left(\boldsymbol{M}\left(\nabla \times \overline{\boldsymbol{A}_{n}}\right), \psi \mu \nabla \times \boldsymbol{A}\right)_{\Omega} \mathrm{d} t \\
= & \int_{0}^{\mathcal{T}}\left(\nabla \times\left[\psi \mu \boldsymbol{M}\left(\nabla \times \overline{\boldsymbol{A}_{n}}\right)\right], \overline{\boldsymbol{A}_{n}}-\boldsymbol{A}\right)_{\Omega} \mathrm{d} t+\int_{0}^{\mathcal{T}}\left(\boldsymbol{M}\left(\nabla \times \overline{\boldsymbol{A}_{n}}\right), \psi \mu \nabla \times \boldsymbol{A}\right)_{\Omega} \mathrm{d} t \\
= & \int_{0}^{\mathcal{T}}\left(\psi \nabla \times\left[\mu \boldsymbol{M}\left(\nabla \times \overline{\boldsymbol{A}_{n}}\right)\right], \overline{\boldsymbol{A}_{n}}-\boldsymbol{A}\right)_{\Omega} \mathrm{d} t+\int_{0}^{\mathcal{T}}\left(\nabla \psi \times\left[\mu \boldsymbol{M}\left(\nabla \times \overline{\boldsymbol{A}_{n}}\right)\right], \overline{\boldsymbol{A}_{n}}-\boldsymbol{A}\right)_{\Omega} \mathrm{d} t \\
& +\int_{0}^{\mathcal{T}}\left(\boldsymbol{M}\left(\nabla \times \overline{\boldsymbol{A}_{n}}\right), \psi \mu \nabla \times \boldsymbol{A}\right)_{\Omega} \mathrm{d} t .
\end{aligned}
$$

We know that $\boldsymbol{A}_{n} \rightarrow \boldsymbol{A}$ in $C\left([0, \mathcal{T}] ; \mathbf{L}^{2}(\boldsymbol{\pi})\right)$ and $\partial_{t} \boldsymbol{A}_{n}$ is bounded in $L^{2}\left((0, \mathcal{T}) ; \mathbf{L}^{2}(\boldsymbol{\pi})\right)$. Therefore also $\overline{\boldsymbol{A}_{n}} \rightarrow \boldsymbol{A}$ in $C\left([0, \mathcal{T}] ; \mathbf{L}^{2}(\boldsymbol{\pi})\right)$. Thus, using $\mu \in H^{1}(\boldsymbol{\pi})$, it is not difficult to see that

$$
\lim _{n \rightarrow \infty} I_{1}=\int_{0}^{\mathcal{T}}(\boldsymbol{p}, \psi \mu \nabla \times \boldsymbol{A})_{\Omega} \mathrm{d} t
$$

Clearly

$$
\begin{aligned}
\lim _{n \rightarrow \infty} I_{2} & =\int_{0}^{\mathcal{T}}(\boldsymbol{M}(\boldsymbol{b}), \psi \mu \nabla \times \boldsymbol{A})_{\Omega} \mathrm{d} t \\
\lim _{n \rightarrow \infty} I_{3} & =\int_{0}^{\mathcal{T}}(\boldsymbol{p}, \psi \mu \boldsymbol{b})_{\Omega} \mathrm{d} t \\
\lim _{n \rightarrow \infty} I_{4} & =\int_{0}^{\mathcal{T}}(\boldsymbol{M}(\boldsymbol{b}), \psi \mu \boldsymbol{b})_{\Omega} \mathrm{d} t .
\end{aligned}
$$

Assembling these auxiliary results we arrive at

$$
\lim _{n \rightarrow \infty} \int_{0}^{\mathcal{T}}\left(\boldsymbol{M}\left(\nabla \times \overline{\boldsymbol{A}_{n}}\right)-\boldsymbol{M}(\boldsymbol{b}), \psi \mu\left(\nabla \times \overline{\boldsymbol{A}_{n}}-\boldsymbol{b}\right)\right)_{\Omega} \mathrm{d} t=\int_{0}^{\mathcal{T}}(\boldsymbol{p}-\boldsymbol{M}(\boldsymbol{b}), \psi \mu(\nabla \times \boldsymbol{A}-\boldsymbol{b}))_{\Omega} \mathrm{d} t \geqslant 0 .
$$


Since $\boldsymbol{b}$ was taken as an arbitrary element of $L^{2}\left((0, \mathcal{T}) ; \mathbf{L}^{2}(\Omega)\right)$ we choose it as $\boldsymbol{b}=\omega \boldsymbol{q}+\nabla \times \boldsymbol{A}$, where $\boldsymbol{q} \in L^{2}\left((0, \mathcal{T}) ; \mathbf{L}^{2}(\Omega)\right)$ and $\omega>0$. Using this substitution in the equation above we obtain

$$
\begin{array}{cl}
\int_{0}^{\mathcal{T}}(\boldsymbol{p}-\boldsymbol{M}(\nabla \times \boldsymbol{A}+\omega \boldsymbol{q}), \mu \psi(-\omega \boldsymbol{q}))_{\Omega} \mathrm{d} t \geqslant 0 & / \cdot \frac{1}{\omega}, \\
\int_{0}^{\mathcal{T}}(\boldsymbol{p}-\boldsymbol{M}(\nabla \times \boldsymbol{A}+\omega \boldsymbol{q}), \mu \psi(-\boldsymbol{q}))_{\Omega} \mathrm{d} t \geqslant 0 \quad & / \omega \rightarrow 0, \\
\int_{0}^{\mathcal{T}}(\boldsymbol{p}-\boldsymbol{M}(\nabla \times \boldsymbol{A}), \mu \psi(-\boldsymbol{q}))_{\Omega} \mathrm{d} t \geqslant 0 \quad / \boldsymbol{q} \text { is arbitrary, hence we choose } \boldsymbol{q}=-\boldsymbol{q}, \\
\int_{0}^{\mathcal{T}}(\boldsymbol{p}-\boldsymbol{M}(\nabla \times \boldsymbol{A}), \mu \psi(-\boldsymbol{q}))_{\Omega} \mathrm{d} t \leqslant 0 .
\end{array}
$$

The conclusion is that $\int_{0}^{\mathcal{T}}(\boldsymbol{p}-\boldsymbol{M}(\nabla \times \boldsymbol{A}), \mu \psi \boldsymbol{q})_{\Omega} \mathrm{d} t=0$ for any non-negative $\psi \in C_{0}^{\infty}(\boldsymbol{\pi})$ and every $\boldsymbol{q} \in$ $L^{2}\left((0, \mathcal{T}) ; \mathbf{L}^{2}(\Omega)\right)$. Hence $\boldsymbol{p}=\boldsymbol{M}(\nabla \times \boldsymbol{A})$ a.e. in $(0, \mathcal{T}) \times \boldsymbol{\pi}$ and $\boldsymbol{M}\left(\nabla \times \overline{\boldsymbol{A}_{n}}\right) \rightarrow \boldsymbol{M}(\nabla \times \boldsymbol{A})$ in $L^{2}\left((0, \mathcal{T}) ; \mathbf{L}^{2}(\boldsymbol{\pi})\right)$.

(iii) Analogously as in (ii) using the strong monotonicity of $\boldsymbol{M}(k 1)$ we conclude

$$
\begin{aligned}
0 & =\lim _{n \rightarrow \infty} \int_{0}^{\mathcal{T}}\left(\boldsymbol{M}\left(\nabla \times \overline{\boldsymbol{A}_{n}}\right)-\boldsymbol{M}(\nabla \times \boldsymbol{A}), \mu \psi\left(\nabla \times \overline{\boldsymbol{A}_{n}}-\nabla \times \boldsymbol{A}\right)\right)_{\Omega} \mathrm{d} t \\
& \geqslant \lim _{n \rightarrow \infty} c_{M} \int_{0}^{\mathcal{T}}\left(\mu \psi,\left|\nabla \times \overline{\boldsymbol{A}_{n}}-\nabla \times \boldsymbol{A}\right|^{2}\right)_{\Omega} \mathrm{d} t \geqslant 0 .
\end{aligned}
$$

Therefore $\lim _{n \rightarrow \infty} \int_{0}^{\mathcal{T}}\left(\mu \psi,\left|\nabla \times \overline{\boldsymbol{A}_{n}}-\nabla \times \boldsymbol{A}\right|^{2}\right)_{\Omega} \mathrm{d} t=0$ for every $0 \leqslant \psi \in C_{0}^{\infty}(\boldsymbol{\pi})$, which implies $\nabla \times \overline{\boldsymbol{A}_{n}} \rightarrow$ $\nabla \times \boldsymbol{A}$ in $L^{2}\left((0, \mathcal{T}) ; \mathbf{L}^{2}(\boldsymbol{\pi})\right)$. Vectorial field $\boldsymbol{M}$ is also Lipschitz continuous, hence $\boldsymbol{M}\left(\nabla \times \overline{\boldsymbol{A}_{n}}\right) \rightarrow \boldsymbol{M}(\nabla \times \boldsymbol{A})$ in $L^{2}\left((0, \mathcal{T}) ; \mathbf{L}^{2}(\boldsymbol{\pi})\right)$ as well.

Now, we are in a position to state our main result.

Theorem 1. Suppose that all assumptions of Proposition 1 are satisfied. Then there exist a solution-triplet $\{\phi, A, u\}$ where $\phi \in L^{2}\left((0, \mathcal{T}) ; H^{1}(T) / \mathbb{R}\right), \boldsymbol{A} \in L^{2}\left((0, \mathcal{T}) ; X_{N, 0}\right)$ with $\partial_{t} \boldsymbol{A} \in L^{2}\left((0, \mathcal{T}) ; \mathbf{L}^{2}(\pi)\right)$ and $u \in C\left([0, \mathcal{T}] ; L^{2}(\pi)\right) \cap$ $L^{\infty}\left((0, \mathcal{T}) ; H_{0}^{1}(\pi)\right)$ with $\partial_{t} u \in L^{2}\left((0, \mathcal{T}) ; L^{2}(\pi)\right)$ and a sub-sequences of $\overline{\phi_{n}}, \boldsymbol{A}_{n}$ and $u_{n}$ (denoted by the same symbol again) such that

(i) $\phi$ and $u$ solve (19)

(ii) $\nabla \overline{\phi_{n}} \rightarrow \nabla \phi$ in $L^{2}\left((0, \mathcal{T}) ; \mathbf{L}^{2}(T)\right)$

(iii) $\phi, u$ and $\boldsymbol{A}$ solve (18)

(iv) $\partial_{t} \boldsymbol{A}_{n} \rightarrow \partial_{t} \boldsymbol{A}$ in $L^{2}\left((0, \mathcal{T}) ; \mathbf{L}^{2}(\pi)\right)$

(v) $\phi, u$ and $A$ solve (20)

holds true for $n \rightarrow+\infty$.

Proof. (i) Existence of a potential $\phi \in H^{1}(T) / \mathbb{R}$ such that $\nabla \overline{\phi_{n}} \rightarrow \nabla \phi$ in $L^{2}\left((0, \mathcal{T})\right.$; $\left.\mathbf{L}^{2}(T)\right)$ follows from the reflexivity of $L^{2}\left((0, \mathcal{T}) ; \mathbf{L}^{2}(T)\right)$. The function $\phi$ has in fact a zero mean over $T$, cf. proof of Lemma 1 .

Take $\xi \in H^{1}(T) / \mathbb{R}$ in (29) and integrate in time

$$
\int_{0}^{\zeta}\left(\overline{\sigma_{\pi_{n}}}(t-\tau) \nabla \overline{\phi_{n}}, \xi\right)_{T} \mathrm{~d} t+\int_{0}^{\zeta}\left(\overline{j_{n}}, \xi\right)_{\Gamma} \mathrm{d} t=0 .
$$

Thanks to Proposition 1(ii) and (v), we pass to the limit for $n \rightarrow \infty$ to get

$$
\int_{0}^{\zeta}\left(\sigma_{\pi}(u) \nabla \phi, \xi\right)_{T} \mathrm{~d} t+\int_{0}^{\zeta}(j, \xi)_{\Gamma} \mathrm{d} t=0 .
$$

Now, differentiating with respect to time, we see that $\phi$ and $u$ solve (19). 
(ii) It holds

$$
\begin{aligned}
0 \leqslant & \sigma_{*} \int_{0}^{\mathcal{T}}\left\|\nabla\left[\overline{\phi_{n}}-\phi\right]\right\|_{L^{2}(T)}^{2} \mathrm{~d} t \\
\leqslant & \int_{0}^{\mathcal{T}}\left(\overline{\sigma_{\pi_{n}}}(t-\tau) \nabla\left[\overline{\phi_{n}}-\phi\right], \nabla\left[\overline{\phi_{n}}-\phi\right]\right)_{T} \mathrm{~d} t \\
= & \int_{0}^{\mathcal{T}}\left(\overline{\sigma_{\pi_{n}}}(t-\tau) \nabla \phi, \nabla \phi\right)_{T} \mathrm{~d} t+\int_{0}^{\mathcal{T}}\left(\overline{\sigma_{\pi_{n}}}(t-\tau) \nabla \overline{\phi_{n}}, \nabla \overline{\phi_{n}}\right)_{T} \mathrm{~d} t \\
& -2 \int_{0}^{\mathcal{T}}\left(\overline{\sigma_{\pi_{n}}}(t-\tau) \nabla \overline{\phi_{n}}, \nabla \phi\right)_{T} \mathrm{~d} t \\
\stackrel{(29)}{=} & \int_{0}^{\mathcal{T}}\left(\overline{\sigma_{\pi_{n}}}(t-\tau) \nabla \phi, \nabla \phi\right)_{T} \mathrm{~d} t-\int_{0}^{\mathcal{T}}\left(\overline{j_{n}}, \overline{\phi_{n}}\right)_{\Gamma} \mathrm{d} t \\
& -2 \int_{0}^{\mathcal{T}}\left(\overline{\sigma_{\pi_{n}}}(t-\tau) \nabla \overline{\phi_{n}}, \nabla \phi\right)_{T} \mathrm{~d} t .
\end{aligned}
$$

Passing to the limit, we conclude

$$
0 \leqslant \lim _{n \rightarrow \infty} \sigma_{*} \int_{0}^{\mathcal{T}}\left\|\nabla\left[\overline{\phi_{n}}-\phi\right]\right\|_{L^{2}(T)}^{2} \mathrm{~d} t \leqslant-\int_{0}^{\mathcal{T}}\left(\sigma_{\pi}(u) \nabla \phi, \nabla \phi\right)_{T} \mathrm{~d} t-\int_{0}^{\mathcal{T}}(j, \phi)_{\Gamma} \mathrm{d} t \stackrel{(i)}{=} 0 .
$$

Therefore, $\nabla \overline{\phi_{n}} \rightarrow \nabla \phi$ in $L^{2}\left((0, \mathcal{T}) ; \mathbf{L}^{2}(T)\right)$.

(iii) We integrate (30) in time

$$
\int_{0}^{\zeta}\left(\overline{\sigma_{\pi_{n}}}(t-\tau) \partial_{t} \boldsymbol{A}_{n}, \boldsymbol{\varphi}\right)_{\pi} \mathrm{d} t+\int_{0}^{\zeta}\left(\mu \boldsymbol{M}\left(\nabla \times \overline{\boldsymbol{A}_{n}}\right), \nabla \times \boldsymbol{\varphi}\right)_{\Omega} \mathrm{d} t+\int_{0}^{\zeta}\left(\overline{\sigma_{\pi_{n}}}(t-\tau) \nabla \overline{\phi_{n}}, \boldsymbol{\varphi}\right)_{T} \mathrm{~d} t=0 .
$$

Using Proposition 1(ii), Proposition 2 and Theorem 1(ii), we pass to the limit for $n \rightarrow \infty$ to see

$$
\int_{0}^{\zeta}\left(\sigma_{\pi}(u) \partial_{t} \boldsymbol{A}, \boldsymbol{\varphi}\right)_{\pi} \mathrm{d} t+\int_{0}^{\zeta}(\mu \boldsymbol{M}(\nabla \times \boldsymbol{A}), \nabla \times \boldsymbol{\varphi})_{\Omega} \mathrm{d} t+\int_{0}^{\zeta}\left(\sigma_{\pi}(u) \nabla \phi, \boldsymbol{\varphi}\right)_{T} \mathrm{~d} t=0 .
$$

Thus, $\phi, u$ and $\boldsymbol{A}$ solve (18).

(iv) The strong convergence of $\nabla \times \overline{\boldsymbol{A}_{n}} \rightarrow \nabla \times \boldsymbol{A}$ in $L^{2}\left((0, \mathcal{T}) ; \mathbf{L}^{2}(\boldsymbol{\pi})\right)$ is guaranteed by Proposition 2(iii). Let us take any $\zeta \in[0, \mathcal{T}]$ such that $\nabla \times \overline{\boldsymbol{A}_{n}}(\zeta) \rightarrow \nabla \times \boldsymbol{A}(\zeta)$ in $\mathbf{L}^{2}(\boldsymbol{\pi})$. This set is dense in $[0, \mathcal{T}]$. Take any non-negative $\psi \in C_{0}^{\infty}(\boldsymbol{\pi})$. We use the positiveness of $\sigma$ to estimate the following

$$
\begin{aligned}
0 \leqslant & \sigma_{*} \int_{0}^{\zeta} \int_{\pi} \psi\left|\partial_{t} \boldsymbol{A}_{n}-\partial_{t} \boldsymbol{A}\right|^{2} \mathrm{~d} x \mathrm{~d} t \leqslant \int_{0}^{\zeta} \int_{\pi} \psi \overline{\sigma_{\pi_{n}}}(t-\tau)\left|\partial_{t} \boldsymbol{A}_{n}-\partial_{t} \boldsymbol{A}\right|^{2} \mathrm{~d} x \mathrm{~d} t \\
= & -2 \int_{0}^{\zeta}\left(\psi \overline{\sigma_{\pi_{n}}}(t-\tau) \partial_{t} \boldsymbol{A}_{n}, \partial_{t} \boldsymbol{A}\right)_{\pi} \mathrm{d} t+\int_{0}^{\zeta}\left(\psi \overline{\sigma_{\pi_{n}}}(t-\tau) \partial_{t} \boldsymbol{A}, \partial_{t} \boldsymbol{A}\right)_{\pi} \mathrm{d} t \\
& +\int_{0}^{\zeta}\left(\psi \overline{\sigma_{\pi_{n}}}(t-\tau) \partial_{t} \boldsymbol{A}_{n}, \partial_{t} \boldsymbol{A}_{n}\right)_{\pi} \mathrm{d} t .
\end{aligned}
$$

We use Lebesgue's dominated convergence theorem combined with Proposition 1(ii) and Proposition 2(i) to pass to the limit for $n \rightarrow \infty$ in the first two terms

$$
\begin{aligned}
& \lim _{n \rightarrow \infty}-2 \int_{0}^{\zeta}\left(\psi \overline{\sigma_{\pi_{n}}}(t-\tau) \partial_{t} \boldsymbol{A}_{n}, \partial_{t} \boldsymbol{A}\right)_{\pi} \mathrm{d} t=-2 \int_{0}^{\zeta}\left(\psi \sigma_{\pi}(u) \partial_{t} \boldsymbol{A}, \partial_{t} \boldsymbol{A}\right)_{\pi} \mathrm{d} t, \\
& \lim _{n \rightarrow \infty} \int_{0}^{\zeta}\left(\psi \overline{\sigma_{\pi_{n}}}(t-\tau) \partial_{t} \boldsymbol{A}, \partial_{t} \boldsymbol{A}\right)_{\pi} \mathrm{d} t=\int_{0}^{\zeta}\left(\psi \sigma_{\pi}(u) \partial_{t} \boldsymbol{A}, \partial_{t} \boldsymbol{A}\right)_{\pi} \mathrm{d} t .
\end{aligned}
$$


We assume that $\zeta \in\left(t_{j-1}, t_{j}\right]$ and use variational formulation (30) to rewrite the third term as

$$
\begin{aligned}
& \int_{0}^{\zeta}\left(\psi \overline{\sigma_{\pi_{n}}}(t-\tau) \partial_{t} \boldsymbol{A}_{n}, \partial_{t} \boldsymbol{A}_{n}\right)_{\pi} \mathrm{d} t=-\int_{0}^{\zeta}\left(\mu \boldsymbol{M}\left(\nabla \times \overline{\boldsymbol{A}_{n}}\right), \nabla \times\left(\psi \partial_{t} \boldsymbol{A}_{n}\right)\right)_{\Omega} \mathrm{d} t \\
& \quad-\int_{0}^{\zeta}\left(\overline{\sigma_{\pi_{n}}}(t-\tau) \nabla \overline{\phi_{n}}, \psi \partial_{t} \boldsymbol{A}_{n}\right)_{T} \mathrm{~d} t \\
&=-\int_{0}^{\zeta}\left(\psi \mu \boldsymbol{M}\left(\nabla \times \overline{\boldsymbol{A}_{n}}\right), \nabla \times \partial_{t} \boldsymbol{A}_{n}\right)_{\Omega} \mathrm{d} t-\int_{0}^{\zeta}\left(\mu \boldsymbol{M}\left(\nabla \times \overline{\boldsymbol{A}_{n}}\right), \nabla \psi \times \partial_{t} \boldsymbol{A}_{n}\right)_{\Omega} \mathrm{d} t \\
& \quad-\int_{0}^{\zeta}\left(\overline{\sigma_{\pi_{n}}}(t-\tau) \nabla \overline{\phi_{n}}, \psi \partial_{t} \boldsymbol{A}_{n}\right)_{T} \mathrm{~d} t \\
&=: R_{1}+R_{2}+R_{3} .
\end{aligned}
$$

Let us rewrite the first term on the RHS and examine it closely

$$
\begin{aligned}
R_{1}= & -\int_{0}^{t_{j}}\left(\psi \mu \boldsymbol{M}\left(\nabla \times \overline{\boldsymbol{A}_{n}}\right), \nabla \times \partial_{t} \boldsymbol{A}_{n}\right)_{\Omega} \mathrm{d} t+\int_{\zeta}^{t_{j}}\left(\psi \mu \boldsymbol{M}\left(\nabla \times \overline{\boldsymbol{A}_{n}}\right), \nabla \times \partial_{t} \boldsymbol{A}_{n}\right)_{\Omega} \mathrm{d} t \\
= & \left.-\sum_{i=1}^{t_{j}} \int_{\Omega} \psi \mu \boldsymbol{M}\left(\nabla \times \boldsymbol{A}_{i}\right) \cdot\left(\nabla \times \boldsymbol{A}_{i}-\nabla \times \boldsymbol{A}_{i-1}\right)\right) \mathrm{d} x \\
& +\int_{\zeta}^{t_{j}}\left(\nabla \times\left(\psi \mu \boldsymbol{M}\left(\nabla \times \overline{\boldsymbol{A}_{n}}\right)\right), \partial_{t} \boldsymbol{A}_{n}\right)_{\Omega} \mathrm{d} t \\
\stackrel{(22)}{\leqslant} & -\sum_{i=1}^{t_{j}} \int_{\Omega} \psi \mu\left\{\Phi_{\boldsymbol{M}}\left(\nabla \times \boldsymbol{A}_{i}\right)-\Phi_{\boldsymbol{M}}\left(\nabla \times \boldsymbol{A}_{i-1}\right)\right\} \mathrm{d} x \\
& +\int_{\zeta}^{t_{j}}\left(\nabla \psi \times\left(\mu \boldsymbol{M}\left(\nabla \times \overline{\boldsymbol{A}_{n}}\right)\right), \partial_{t} \boldsymbol{A}_{n}\right)_{\Omega} \mathrm{d} t+\int_{\zeta}^{t_{j}}\left(\psi \nabla \times\left(\mu \boldsymbol{M}\left(\nabla \times \overline{\boldsymbol{A}_{n}}\right)\right), \partial_{t} \boldsymbol{A}_{n}\right)_{\Omega} \mathrm{d} t \\
= & -\int_{\Omega}^{t_{j}} \psi \mu \Phi_{\boldsymbol{M}}\left(\nabla \times \boldsymbol{A}_{j}\right) \mathrm{d} x+\int_{\Omega} \psi \mu \Phi_{\boldsymbol{M}}\left(\nabla \times \boldsymbol{A}_{0}\right) \mathrm{d} x \\
& +\int_{\zeta}^{t_{j}}\left(\nabla \psi \times\left(\mu \boldsymbol{M}\left(\nabla \times \overline{\boldsymbol{A}_{n}}\right)\right), \partial_{t} \boldsymbol{A}_{n}\right)_{\Omega} \mathrm{d} t+\int_{\zeta}^{t_{j}}\left(\psi \nabla \times\left(\mu \boldsymbol{M}\left(\nabla \times \overline{\boldsymbol{A}_{n}}\right)\right), \partial_{t} \boldsymbol{A}_{n}\right)_{\Omega} \mathrm{d} t \\
= & -\int_{\Omega}^{t_{j}} \psi \mu \Phi_{\boldsymbol{M}}\left(\boldsymbol{M}\left(\nabla \times \overline{\boldsymbol{A}_{n}}(\zeta)\right) \mathrm{d} x+\int_{\Omega} \psi \mu \Phi_{\boldsymbol{M}}\left(\nabla \times \boldsymbol{A}_{0}\right) \mathrm{d} x\right. \\
& +\int_{\zeta}^{t_{j}}\left(\nabla \psi \times\left(\mu \boldsymbol{M}\left(\nabla \times \overline{\boldsymbol{A}_{n}}\right)\right), \partial_{t} \boldsymbol{A}_{n}\right)_{\Omega} \mathrm{d} t+\int_{\zeta}^{t_{j}}\left(\psi \nabla \times\left(\mu \boldsymbol{M}\left(\nabla \times \overline{\boldsymbol{A}_{n}}\right)\right), \partial_{t} \boldsymbol{A}_{n}\right)_{\Omega} \mathrm{d} t .
\end{aligned}
$$

Now, we are able to pass to the limit for $n \rightarrow \infty$ to find

$$
\begin{aligned}
& \lim _{n \rightarrow \infty} R_{2}=-\int_{0}^{\zeta}\left(\mu \boldsymbol{M}(\nabla \times \boldsymbol{A}), \nabla \psi \times \partial_{t} \boldsymbol{A}\right)_{\Omega} \mathrm{d} t, \\
& \lim _{n \rightarrow \infty} R_{3}=-\int_{0}^{\zeta}\left(\sigma_{\pi}(u) \nabla \phi, \psi \partial_{t} \boldsymbol{A}\right)_{T} \mathrm{~d} t,
\end{aligned}
$$

and

$$
\begin{aligned}
\lim _{n \rightarrow \infty} R_{1} & \leqslant-\int_{\Omega} \psi \mu \Phi_{\boldsymbol{M}}(\nabla \times \boldsymbol{A}(\zeta)) \mathrm{d} x+\int_{\Omega} \psi \mu \Phi_{\boldsymbol{M}}(\nabla \times \boldsymbol{A}(0)) \mathrm{d} x \\
& =-\int_{0}^{\zeta} \int_{\Omega} \psi \mu \frac{\mathrm{d} \Phi_{\boldsymbol{M}}(\nabla \times \boldsymbol{A})}{\mathrm{d} t} \mathrm{~d} x \mathrm{~d} t \\
& =-\int_{0}^{\zeta} \int_{\Omega} \psi \mu \boldsymbol{M}(\nabla \times \boldsymbol{A}) \cdot \partial_{t}(\nabla \times \boldsymbol{A}) \mathrm{d} x \mathrm{~d} t \\
& =-\int_{0}^{\zeta}\left(\mu \boldsymbol{M}(\nabla \times \boldsymbol{A}), \psi \nabla \times\left(\partial_{t} \boldsymbol{A}\right)\right)_{\Omega} \mathrm{d} t
\end{aligned}
$$


Thus

$$
\begin{aligned}
& \lim _{n \rightarrow \infty} R_{1}+R_{2}+R_{3} \leqslant-\int_{0}^{\zeta}\left(\mu \boldsymbol{M}(\nabla \times \boldsymbol{A}), \nabla \times\left(\psi \partial_{t} \boldsymbol{A}\right)\right)_{\Omega} \mathrm{d} t-\int_{0}^{\zeta}\left(\sigma_{\pi}(u) \nabla \phi, \psi \partial_{t} \boldsymbol{A}\right)_{T} \mathrm{~d} t \\
& \stackrel{(18)}{=} \int_{0}^{\zeta}\left(\psi \sigma_{\pi}(u) \partial_{t} \boldsymbol{A}, \partial_{t} \boldsymbol{A}\right)_{\pi} \mathrm{d} t .
\end{aligned}
$$

Thus, collecting all estimates above, we see that

$$
0 \leqslant \lim _{n \rightarrow \infty} \int_{0}^{\zeta} \int_{\pi} \psi\left|\partial_{t} \boldsymbol{A}_{n}-\partial_{t} \boldsymbol{A}\right|^{2} \mathrm{~d} x \mathrm{~d} t \leqslant 0
$$

Please note that this is valid for any non-negative $\psi \in C_{0}^{\infty}(\boldsymbol{\pi})$. Since the set of $\zeta \in[0, \mathcal{T}]$ for which $\nabla \times \overline{\boldsymbol{A}_{n}}(\zeta) \rightarrow$ $\nabla \times \boldsymbol{A}(\zeta)$ in $\mathbf{L}^{2}(\Omega)$ is dense in $[0, \mathcal{T}]$, we achieve a strong convergence of $\partial_{t} \boldsymbol{A}_{n}$ in $L^{2}\left((0, \mathcal{T}) ; \mathbf{L}^{2}(\pi)\right)$ i.e. $\partial_{t} \boldsymbol{A}_{n} \rightarrow \partial_{t} \boldsymbol{A}$ in $L^{2}\left((0, \mathcal{T}) ; \mathbf{L}^{2}(\pi)\right)$.

(v) Take $\psi \in H^{1}(\pi)$ in (31) and integrate in time

$$
\begin{gathered}
\left(\overline{\beta_{n}}(t)-\beta_{n}(0), \psi\right)_{\pi}+\left(\beta_{n}(t)-\overline{\beta_{n}}(t), \psi\right)_{\pi}+\int_{0}^{t}\left(\overline{\lambda_{n}} \nabla \overline{u_{n}}, \nabla \psi\right)_{\pi} \mathrm{d} s \\
=\int_{0}^{t}\left(\mathcal{R}_{r}\left(\overline{\sigma_{\pi_{n}}}(s-\tau)\left|\partial_{t} \boldsymbol{A}_{n}+\chi_{T} \nabla \overline{\phi_{n}}\right|^{2}\right), \psi\right)_{\pi} \mathrm{d} s .
\end{gathered}
$$

Using Lebesgue's dominated convergence theorem, together with Proposition 1(ii), Theorem 1(ii) and (iv) enables passing to the limit for $n \rightarrow \infty$ in the RHS of the equation above

$$
\begin{gathered}
\lim _{n \rightarrow \infty} \int_{0}^{t}\left(\mathcal{R}_{r}\left(\overline{\sigma_{\pi_{n}}}(s-\tau)\left|\partial_{t} \boldsymbol{A}_{n}+\chi_{T} \nabla \overline{\phi_{n}}\right|^{2}\right), \psi\right)_{\pi} \mathrm{d} s \\
=\int_{0}^{t}\left(\mathcal{R}_{r}\left(\sigma_{\pi}(u)\left|\partial_{t} \boldsymbol{A}+\chi_{T} \nabla \phi\right|^{2}\right), \psi\right)_{\pi} \mathrm{d} s .
\end{gathered}
$$

Proposition 1 let us pass to the limit for $n \rightarrow \infty$ on the LHS. Note that term $\left(\beta_{n}(t)-\overline{\beta_{n}}(t), \psi\right)_{\pi}$ vanishes since $\lim _{n \rightarrow \infty}\left(\beta_{n}(t)-\overline{\beta_{n}}(t), \psi\right)_{\pi}=0$ for every $t \in[0, \mathcal{T}]$. Therefore gathering all results above brings us to

$$
(\beta(u(t))-\beta(u(0)), \psi)_{\pi}+\int_{0}^{t}(\lambda \nabla u, \nabla \psi)_{\pi} \mathrm{d} s=\int_{0}^{t}\left(\mathcal{R}_{r}\left(\sigma_{\pi}(u)\left|\partial_{t} \boldsymbol{A}+\chi_{T} \nabla \phi\right|^{2}\right), \psi\right)_{\pi} \mathrm{d} s .
$$

The only thing left to be done to finish the proof is differentiating with respect to time. Thus, we see that $\phi, u$ and $\boldsymbol{A}$ indeed solve (20).

\section{Numerical simulation}

To support our proposed numerical scheme (28) obtained from the variational formulation (27), (26), (25) we provide a numerical simulation of induction hardening process. The domain used in the simulation is reported in Fig. 4. This domain is more complex than its simplified version in Fig. 1, but our theoretical results for this type hold regardless, because the inclusion $\boldsymbol{X}_{N, 0} \subset \mathbf{H}^{1}(\Omega)$ holds true also for convex domains (without a smooth boundary), cf. [21, Theorem 2.17]. Since we want our simulation to be realistic we use physical constants. Unknown functions representing nonlinearities are chosen accordingly to satisfy (21)

$$
\begin{aligned}
& \sigma_{\pi}(u)=2 \sigma_{c}+\sigma_{c}\left(2-\left(1+\frac{1}{1+u}\right)^{1+u}\right), \\
& \beta(u)=\beta_{c} \sqrt{u}, \\
& \boldsymbol{M}(\nabla \times \boldsymbol{A})=\left(1+e^{-|\nabla \times \boldsymbol{A}|}\right) \nabla \times \boldsymbol{A}, \\
& \sigma_{c}, \beta_{c}, \mu, \lambda \Longrightarrow \text { Physical constants, } \\
& \mathcal{T}=0.02, \quad \boldsymbol{A}_{0}=\mathbf{0}, \quad u_{0}=293 \text { Kelvin. }
\end{aligned}
$$

We split the time interval $[0, \mathcal{T}]$ in 1280 equidistant parts $\left(\tau=1.5625 \mathrm{e} 10^{-5}\right)$ and use the open source finite element environment Gmsh/GetDP [32,33], freely available online on http://www.onelab.info, to solve the system (26), (25) 

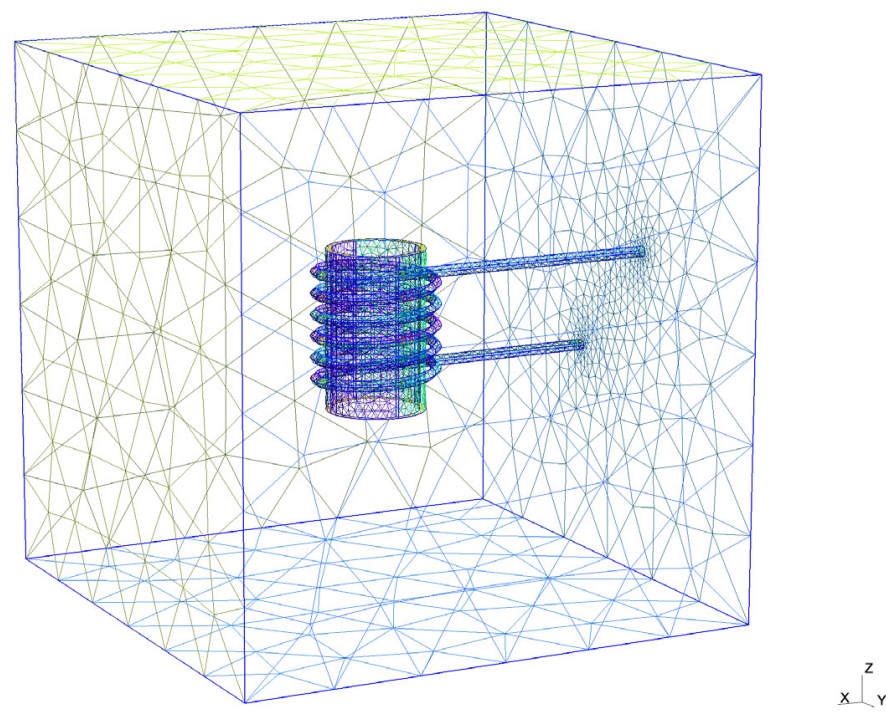

Fig. 4. Meshed domain.

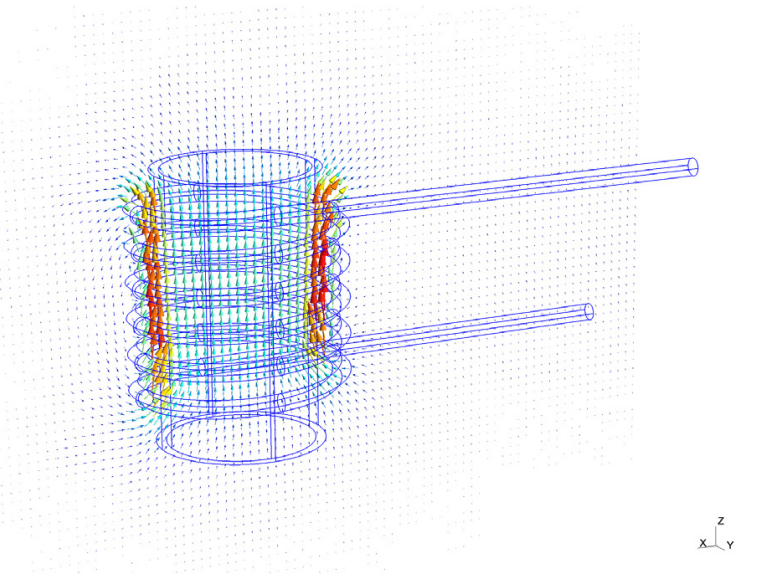

(a) Magnetic induction field.

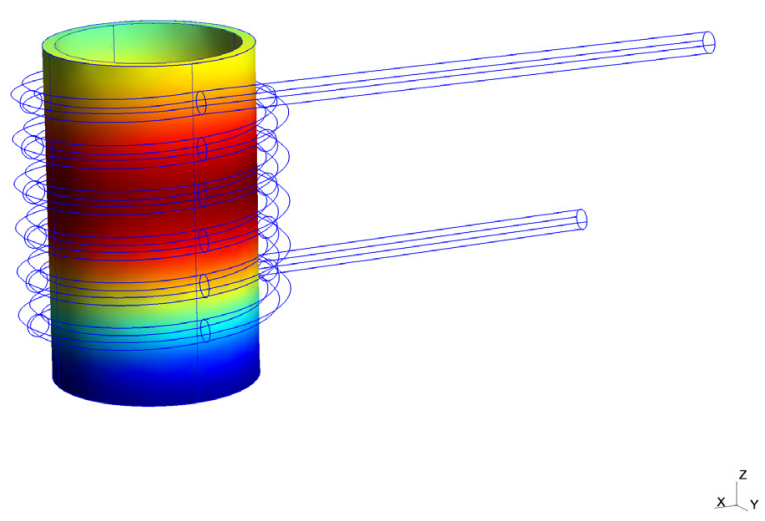

(b) Temperature

Fig. 5. Reference solutions in time $t=0.015$.

and (27) at each time step, after spatial discretization using Whitney finite elements on tetrahedra (edge elements for the magnetic vector potential, nodal elements for the electric scalar potential and the temperature) [34]. The Neumann boundary condition in (14) and (17) is simply treated by adding the corresponding surface term arising from integration by parts in the weak formulation. The mesh contained 26765 tetrahedra, leading to a total of 29714 unknowns. We denote obtained solutions for the magnetic induction field and the temperature function as reference solutions $\boldsymbol{b}_{r e f}$ and $u_{r e f}$, respectively. Typical solutions are plotted in Fig. 5.

To show that our scheme is converging to $\boldsymbol{b}_{r e f}$ and $u_{r e f}$ we compute other numerical solutions for number of time steps 10, 20, 40, 80, 160, 320 and 640 and compare them with $\boldsymbol{b}_{r e f}$ and $u_{r e f}$. We analyze these solutions in certain measurement points of our domain (see Fig. 6) and at certain time steps, namely $t_{i}=0.002 i$, where $i=1, \ldots, 10$. Relative errors of a given numerical solution $\boldsymbol{b}_{n}$ from the reference solution $\boldsymbol{b}_{r e f}$ and $u_{n}$ from $u_{r e f}$ are then calculated 


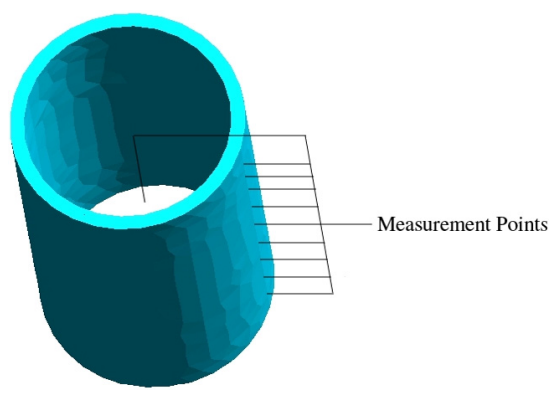

Fig. 6. Measurement points.

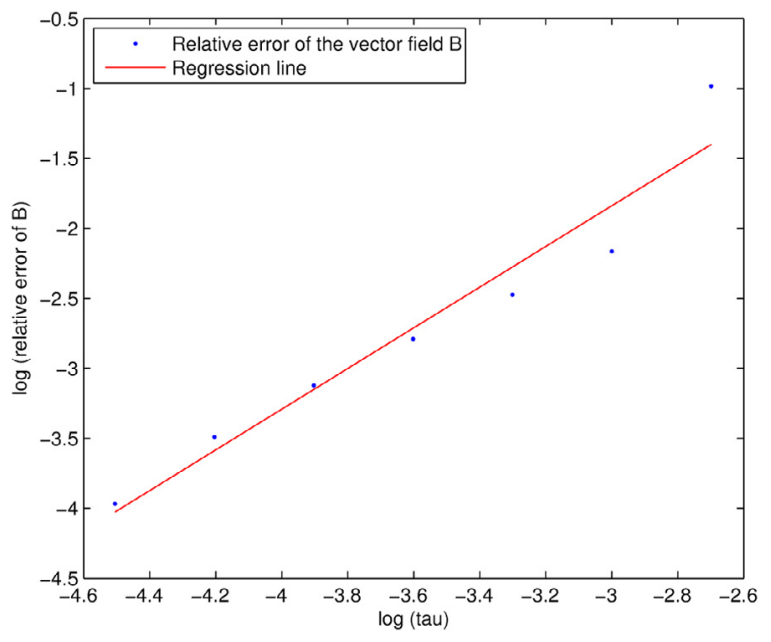

(a) Relative error of the magnetic induction field $\boldsymbol{B}$ with respect to a decreasing time step $\tau$.

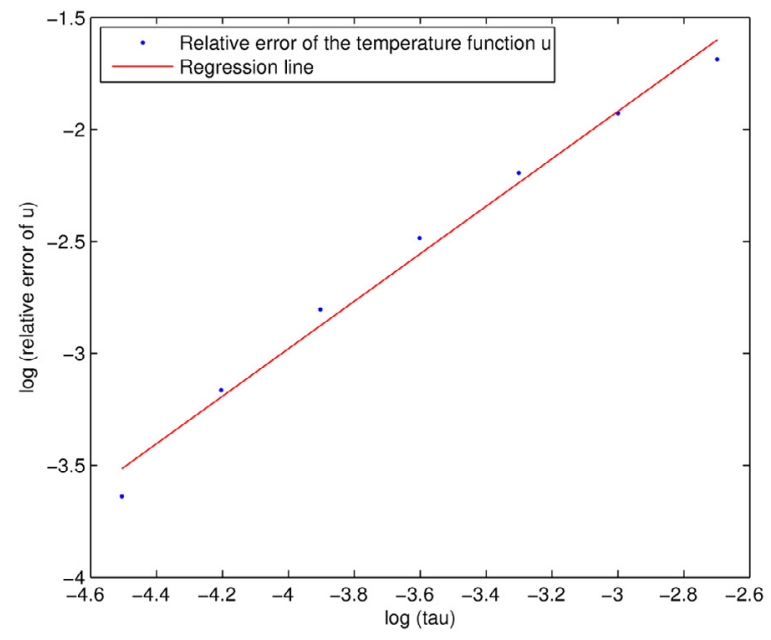

(b) Relative error of the temperature function $u$ with respect to a decreasing time step $\tau$.

Fig. 7. Logarithmically scaled plot of a decreasing time step $\tau$ and the relative errors.

in the following manner

$$
\begin{gathered}
\left|\boldsymbol{b}_{r e f}\right|=\sum_{P_{j} \in P} \sum_{i=1}^{10}\left|\boldsymbol{b}_{r e f}\left(P_{j}, t_{i}\right)\right| \quad\left|u_{r e f}\right|=\sum_{P_{j} \in P} \sum_{i=1}^{10}\left|u_{r e f}\left(P_{j}, t_{i}\right)\right| \\
\left|\boldsymbol{b}_{r e f}-\boldsymbol{b}_{n}\right|=\sum_{P_{j} \in P} \sum_{i=1}^{10}\left|\boldsymbol{b}_{r e f}\left(P_{j}, t_{i}\right)-\boldsymbol{b}_{n}\left(P_{j}, t_{i}\right)\right|\left|u_{r e f}-u_{n}\right|=\sum_{P_{j} \in P} \sum_{i=1}^{10}\left|u_{r e f}\left(P_{j}, t_{i}\right)-u_{n}\left(P_{j}, t_{i}\right)\right| \\
\operatorname{rel} \boldsymbol{b}_{n}=\frac{\left|\boldsymbol{b}_{r e f}-\boldsymbol{b}_{n}\right|}{\left|\boldsymbol{b}_{r e f}\right|} \operatorname{rel} u_{n}=\frac{\left|u_{r e f}-u_{n}\right|}{\left|u_{r e f}\right|},
\end{gathered}
$$

where $P$ is the set of measurement points. Please bear in mind that the index $n$ refers to the numerical solution computed on a mesh with $2^{n-1} \cdot 10$ time steps. The evolution of these errors with increasing number of time steps can be seen in Fig. 7.

If the error of a given numerical solution $f_{\tau}$ from the exact solution $f$ depends smoothly on a time step $\tau$ then there exist an error coefficient $D$ such that

$$
f_{\tau}-f=D \tau^{p}+\mathcal{O}\left(\tau^{p+1}\right)
$$


where $p$ represents the order of convergence. Using the fact that the difference of $f_{\tau}-f_{\tau / 2}$ decays to zero with the same speed as $f_{\tau}-f$ we can estimate the order of convergence without knowing the exact solution $f$, i.e.

$$
\frac{f_{\tau}-f_{\tau / 2}}{f_{\tau / 2}-f_{\tau / 4}}=\frac{D \tau^{p}-D(\tau / 2)^{p}+\mathcal{O}\left(\tau^{p+1}\right)}{D(\tau / 2)^{p}-D(\tau / 4)^{p}+\mathcal{O}\left(\tau^{p+1}\right)}=2^{p}+\mathcal{O}(\tau)
$$

which gives us

$$
\log _{2}\left(\frac{f_{\tau}-f_{\tau / 2}}{f_{\tau / 2}-f_{\tau / 4}}\right)=p+\mathcal{O}(\tau)
$$

Applying the formula above to our numerical solutions we obtain an estimation for the order of convergence of $\boldsymbol{b}_{n}$ and $u_{n}$

$$
p_{u} \approx 0.9830 \quad \text { and } \quad p_{b} \approx 1.0010 .
$$

This provides a strong indication that the convergence of our numerical scheme is linear.

\section{Conclusion}

We have provided a derivation of a mathematical model of induction hardening process with inclusion of a nonlinear relation between the magnetic field and the magnetic induction field. We have also proven an existence of a weak solution of our model.

To support the theoretical results we have coded the numerical scheme implied by a variational formulation and ran few simulations. However, we did not have an analytic solution. Numerical solutions are therefore compared with a numerical reference one computed on a fine reference mesh. Afterwards we have investigated how the numerical solutions computed for the increasing number of time steps (starting at 10) were behaving according to the reference solutions $\boldsymbol{b}_{r e f}$ and $u_{r e f}$. We have obtained an improving match with increasing number of time steps. Since we do not have a proof of a unique solution of our model we could not prove the convergence of the scheme rigorously. However the numerical experiments suggest that the scheme might really be convergent.

In the following work we would like to provide a proof of a unique solution. The coupling between the vector potential equation and the heat equation in the form of the temperature dependent function $\sigma(u)$ prevents us from obtaining the desired energy estimates needed to prove the uniqueness of the solution and therefore it still remains an open problem.

\section{Acknowledgments}

J. Chovan was supported by the BOF-project no. 01J04113, Ghent University, Belgium. Ch. Geuzaine and M. Slodička were partially supported by the IAP P7/02-project of the Belgian Science Policy.

\section{References}

[1] C.M. Elliott, S. Larsson, A finite element model for the time-dependent Joule heating problem, Math. Comp. 64 (212) (1995) $1433-1453$.

[2] G. Akrivis, S. Larsson, Linearly implicit finite element methods for the time-dependent Joule heating problem, BIT 45 (2005) $429-442$.

[3] J. Barglik, I. Doležel, P. Karban, B. Ulrych, Modelling of continual induction hardening in quasi-coupled formulation, Compel 24 (1) (2005) $251-260$

[4] A. Bermúdez, D. Gómez, M. Muñiz, P. Salgado, Transient numerical simulation of a thermoelectrical problem in cylindrical induction heating furnaces, Adv. Comput. Math. 26 (1-3) (2007) 39-62.

[5] D. Sun, V. Manoranjan, H.-M. Yin, Numerical solutions for a coupled parabolic equations arising induction heating processes, Discrete Contin. Dyn. Syst. 2007 (2007) 956-964.

[6] H. Gao, Optimal error analysis of Galerkin fems for nonlinear Joule heating equations, J. Sci. Comput. 58 (3) (2014) $627-647$.

[7] H.-M. Yin, Global solutions of Maxwell's equations in an electromagnetic field with a temperature-dependent electrical conductivity, European J. Appl. Math. 5 (1994) 57-64.

[8] H.-M. Yin, On Maxwell's equations in an electromagnetic field with the temperature effect, SIAM J. Math. Anal. 29 (3) (1998) 637-651.

[9] H.-M. Yin, Regularity of weak solution to Maxwell's equations and applications to microwave heating, J. Differential Equations 200 (1) (2004) 137-161.

[10] D. Sun, V. Manoranjan, H.-M. Yin, Numerical solutions for a coupled parabolic equations arising induction heating processes, Dyn. Syst. (2007) 956-964.

[11] M. Bień, Global solutions of the non-linear problem describing Joule’s heating in three space dimensions, Math. Methods Appl. Sci. 28 (9) (2005) 1007-1030. 
[12] D. Hömberg, A mathematical model for induction hardening including mechanical effects, Nonlinear Anal. RWA 5 (1) (2004) 55-90.

[13] D. Hömberg, T. Petzold, E. Rocca, Analysis and simulations of multifrequency induction hardening, Nonlinear Anal. RWA 22 (0) (2015) 84-97.

[14] A. Bossavit, J.F. Rodrigues, On the electromagnetic "induction heating" problem in bounded domains, Adv. Math. Sci. Appl. 4 (1) (1994) 79-92.

[15] J. Chovan, M. Slodička, Induction hardening of steel with restrained joule heating and nonlinear law for magnetic induction field: Solvability, JCAM 311 (2017) 630-644.

[16] M. Slodička, J. Chovan, Solvability for induction hardening including nonlinear magnetic field and controlled joule heating, Appl. Anal. Available online at http://dx.doi.org/101080/0003681120161243661.

[17] L.D. Landau, E.M. Lifshitz, Electrodynamics of Continuous Media, Vol. 8, Oxford: Pergamon Press, 1960.

[18] M. Vajnberg, Variational Method and Method of Monotone Operators in the Theory of Nonlinear Equations, John Wiley \& Sons, 1973.

[19] V. Girault, P.-A. Raviart, Finite Element Methods for Navier-Stokes Equations, Springer, Berlin, 1986.

[20] P. Monk, Finite Element Methods for Maxwell's Equations, Oxford University Press Inc., New York, 2003.

[21] C. Amrouche, C. Bernardi, M. Dauge, V. Girault, Vector potentials in three-dimensional non-smooth domains, Math. Methods Appl. Sci. 21 (9) (1998) 823-864.

[22] R.A. Adams, Sobolev Spaces, Academic Press, Springer Verlag, 1978.

[23] J. Kačur, Method of Rothe in Evolution Equations, in: Teubner Texte zur Mathematik, vol. 80, Teubner, Leipzig, 1985.

[24] K. Rektorys, The Method of Discretization in Time and Partial Differential Equations, in: Mathematics and Its Applications (East European Series), vol. 4, D. Reidel Publishing Company, Dordrecht - Boston - London, 1982.

[25] J. Nečas, Introduction to the Theory of Nonlinear Elliptic Equations, John Wiley \& Sons Ltd, Chichester, 1986.

[26] M. Slodička, S. Dehilis, A nonlinear parabolic equation with a nonlocal boundary term, J. Comput. Appl. Math. 233 (12) (2010) $3130-3138$.

[27] T. Roubíček, Nonlinear Partial Differential Equations with Applications, Birkhäuser, Berlin, 2005.

[28] G.J. Minty, On a "monotonicity" method for the solution of nonlinear equations in Banach spaces, Proc. Natl. Acad. Sci. 50 (6) (1963) $1038-1041$

[29] F.E. Browder, Nonlinear elliptic boundary value problems. ii, Trans. Amer. Math. Soc. 117 (1965) 530-550.

[30] A. Kufner, O. John, S. Fučík, Function Spaces, Monographs and Textbooks on Mechanics of Solids and Fluids, Noordhoff International Publishing, Leyden, 1977.

[31] L.C. Evans, Partial Differential Equations, Vol. 19, American Mathematical Society, RI, 1998.

[32] C. Geuzaine, J.-F. Remacle, Gmsh: a three-dimensional finite element mesh generator with built-in pre- and post-processing facilities, Internat. J. Numer. Methods Engrg. 79 (11) (2009) 1309-1331.

[33] P. Dular, C. Geuzaine, F. Henrotte, W. Legros, A general environment for the treatment of discrete problems and its application to the finite element method, IEEE Trans. Magn. 34 (5) (1998) 3395-3398.

[34] A. Bossavit, Computational electromagnetism, in: Variational Formulations, Complementarity, Edge Elements, in: Electromagnetism, vol. 18, Academic Press, Orlando, FL, 1998. 